\title{
Geographic-Based Spray-and-Relay (GSaR): An Efficient Routing Scheme for DTNs
}

\author{
Yue Cao, Zhili Sun, Member, IEEE, Ning Wang, Member, IEEE, Maryam Riaz, \\ Haitham Cruickshank, Member, IEEE, and Xiulei Liu
}

\begin{abstract}
In this paper, we design and evaluate the proposed geographic-based spray-and-relay (GSaR) routing scheme in delay/disruption-tolerant networks. To the best of our knowledge, GSaR is the first spray-based geographic routing scheme using historical geographic information for making a routing decision. Here, the term spray means that only a limited number of message copies are allowed for replication in the network. By estimating a movement range of destination via the historical geographic information, GSaR expedites the message being sprayed toward this range, meanwhile prevents that away from and postpones that out of this range. As such, the combination of them intends to fast and efficiently spray the limited number of message copies toward this range and effectively spray them within range, to reduce the delivery delay and increase the delivery ratio. Furthermore, GSaR exploits delegation forwarding to enhance the reliability of the routing decision and handle the local maximum problem, which is considered to be the challenges for applying the geographic routing scheme in sparse networks. We evaluate GSaR under three city scenarios abstracted from real world, with other routing schemes for comparison. Results show that GSaR is reliable for delivering messages before the expiration deadline and efficient for achieving low routing overhead ratio. Further observation indicates that GSaR is also efficient in terms of a low and fair energy consumption over the nodes in the network.
\end{abstract}

Index Terms-Delay/disruption-tolerant networks (DTNs), efficiency, geographic routing, spraying messages.

\section{INTRODUCTION}

$\mathbf{R}$ ESEARCH into mobile ad hoc networks (MANETs) often assumes the contemporaneous end-to-end connectivity, which inevitably poses challenges for relaying messages in the challenged wireless networks, suffering from frequent disruption, sparse network density, and limited capability of devices. With this in mind, delay/disruption-tolerant networks (DTNs) [1] have received great interest from the research community and are envisioned for many terrestrial applications. Apart from interplanetary networks [2], other examples of

Manuscript received October 15, 2013; revised May 20, 2014; accepted May 31, 2014. Date of publication June 17, 2014; date of current version April 14, 2015. This work was supported in part by the EU FP7 Mission project, the EU FP7 C-DAX project, and the China Academy of Telecommunication Technology under Grant No-2007-DQ-305156. The review of this paper was coordinated by Dr. N.-D. Dao.

Y. Cao, Z. Sun, N. Wang, M. Riaz, and H. Cruickshank are with the Center for Communication Systems Research, University of Surrey, Guildford GU2 7XH, U.K. (e-mail: Y.Cao@surrey.ac.uk; Z.Sun@surrey.ac.uk; N.Wang@ surrey.ac.uk; M.Riaz@surrey.ac.uk; H.Cruickshank@surrey.ac.uk).

$\mathrm{X}$. Liu is with the National Engineering Laboratory for Disaster Backup and Recovery, Beijing University of Posts and Telecommunications, Beijing 100876, China (e-mail: xiuleiliu@hotmail.com).

Color versions of one or more of the figures in this paper are available online at http://ieeexplore.ieee.org.

Digital Object Identifier 10.1109/TVT.2014.2331395
DTNs include sparse vehicular ad hoc networks [3], ocean sensor networks [4], and pocket switched networks [5].

Here, the intermittent connectivity in these networks can be a result of mobility, energy, wireless range, and sparse network density. For example, if the encounter opportunity is unavailable, the node may store messages in its buffer and carry them until a new connectivity for relaying these messages is available, which is known as the store-carry-forward (SCF) routing behavior. This inherent uncertainty about the network topology makes routing in DTNs a challenging problem.

Geographic routing, in general, requires that each node knows its own location and the location of its destination. Different from topology-based routing, geographic routing exploits the geographic information instead of topological connectivity information to relay messages, to gradually approach and eventually reach the intended destination. Although numerous previous works have been proposed in designing a powerful routing scheme using historical topology information, geographic routing in DTNs has not received much attention, as reviewed in [1].

In spite that geographic routing does not rely on the varied network topology information to relay messages, the following three challenges should be addressed if applying this scheme in DTNs.

- Regarding geographic routing in MANETs, messages can be greedily relayed toward the destination via the continuously connected path in a short time. However, in DTNs, the node that is currently closer to the destination may not be so in the future. This is because the node moving away from the destination may not encounter other nodes in a short time, when considering nodal mobility and sparse network density.

- In MANETs, the local maximum problem ${ }^{1}$ [6] implies that the message cannot be relayed with a positive geometric progress toward the destination. Here, for candidate node ${ }^{2}$ selection in DTNs, the utility metric is defined according to the historical information to qualify the encountered node. However, conventional approaches designed

\footnotetext{
${ }^{1}$ This problem implies that if a better relay node is unavailable, the message carrier will keep on carrying its message. In light of this, the message delivery is delayed or even degraded if a better relay node is never met. Using the distance metric as an example, any node closer to the destination is qualified with a better delivery potential. However, a message cannot be relayed if any encountered node is farther away from the destination.

${ }^{2}$ For the purpose of generalization, the candidate node is the relay node of the next hop, which is selected based on a criterion that makes positive effort for message delivery.
} 
for MANETs rely on the high network density, which is infeasible in DTNs due to sparse network density. In this case, the message delivery will be delayed because of the insufficient number of available encountered nodes for handling this problem.

- Considering the mobility of the destination, this challenge limits the feasibility of using a centralized location service system to distribute the real-time geographic information in sparse networks. This happens due to the fact that there is a long delay to request/reply the real-time location information in DTNs; thus, the obtained information may be outdated and inaccurate for routing decision.

In the literature, replicating message copies is effective in increasing the delivery ratio in sparse networks, for which these replicated message copies increase the diffusion speed and the possibility that one of them would be delivered. In general, there are two ways to reduce replication redundancy: On one hand, previous works [7]-[11] replicate messages to any betterqualified candidate node. On the other hand, the spray-based ${ }^{3}$ routing schemes [12]-[14] reduce the replication redundancy by limiting the number of message copies in the network. Compared with those in the former branch, the schemes in the latter branch inherently assume that nodal mobility is more sufficient for message delivery.

In this paper, the proposed geographic-based spray-and-relay (GSaR) is characterized into the latter branch, by delivering messages given the limited number of replications. Here, the message replication is controlled by a copy ticket cached in each message, where the initial value of this copy ticket is predefined based on a scenario and distributed to the selected candidate node.

1) Given the historical location, moving speed, and encounter time recorded in the past, the movement range of the destination is estimated by referring to [15].

2) Here, based on the nature of the spray-based routing scheme, our intention is threefold.

- Expedite message copies being sprayed toward this range to reduce delivery delay.

- Prevent message copies being sprayed away from this range to reduce routing overhead.

- Postpone message copies being sprayed out of this range to increase delivery probability.

As a backup scheme, if the given historical geographic information of the destination is unavailable, messages are sprayed considering the relative moving direction between pairwise nodes and their moving speed.

3) The local maximum problem has not been adequately addressed by other researchers in the literature, particularly considering the limited number of message copies. Since this problem will delay message delivery, we propose to continually spray message copies, considering that the candidate node is unable to achieve delivery before the message expiration deadline.

\footnotetext{
${ }^{3}$ Assuming $L$ is a predefined value, each message can only be replicated for $(L-1)$ times. In general, the value of $L$ is quite small compared with the total number of nodes in the network.
}

4) Delegation forwarding (DF) [16] is investigated for overcoming the limitation of a routing decision, meanwhile enhancing the scheme for handling the local maximum problem. Here, the motivation for using DF overcomes the limitation if pairwise encountered nodes are with inconsistent status, such as when one of them is out of the movement range estimated for the destination while another one is within this range. Furthermore, using DF intends to select the candidate node with the historically best delivery potential rather than that with a currently better delivery potential. This is important particularly when the number of message replications is limited, as GSaR only allows a limited number of nodes to carry messages.

5) Apart from the design of the routing framework, messages are under prioritized transmission and deletion, by taking into account the limited bandwidth and buffer space. Since a message may expire before its candidate node encounters the destination, GSaR allocates the bandwidth and buffer space for the message that could be delivered within the expiration deadline, considering the mobility of the candidate node and awareness of the destination's historical geographic information.

For performance evaluation, GSaR is compared with other routing schemes [7], [8], [12], [13], [17] under three city scenarios, namely, Helsinki, Tokyo, and San Francisco. Furthermore, we examine the energy consumption of GSaR accounted for transmission, to show its fairness. Simulation results show that $\mathrm{GSaR}$ outperforms the compared routing schemes, in terms of lower overhead ratio while maintaining high delivery ratio. It also consumes a low and fair energy consumption over the nodes in the network.

The remainder of this paper is as follows. The related work is presented in Section II. Following the assumption and overview presented in Section III, the design of GSaR is detailed in Section IV and evaluated in Section V, respectively. Finally, the conclusion is given in Section VI.

\section{RElated WORK}

\section{A. Replication-Based Routing Schemes}

The schemes in this branch do not limit the number of copies of each message that can be replicated in the network. The benchmark scheme, namely, Epidemic [18], floods message copies to any node in the network. In spite that Epidemic achieves the highest delivery ratio, a huge network resource including bandwidth and buffer space are wasted for replication redundancy. Here, using the utility metric to qualify the nodal delivery potential for controlling replication has been studied by previous works.

1) Based on Historical Encounter Information: In Probabilistic ROuting Protocol using History of Encounters and Transitivity (PROPHET) [17], the utility metric is based on an encounter probability. The powerful Resource Allocation Protocol for Intentional DTN (RAPID) [19] treats the routing problem as a resource allocation aspect, where the utility metric is estimated as the remaining delivery delay. To reduce 
replication redundancy, DF [16] is proposed to optimize the candidate node selection, using the topology-based utility metric.

2) Based on Geographic Information: As an alternative scheme to those using topology-based historical encounter information, distance-aware epidemic routing (DAER) [9] adopts current distance toward the destination as the utility metric using real-time location information. Upon the design of DAER, packet-oriented routing [11] takes into account the distance factor for all the requested messages. Different from those considering stationary destination [10], [20], [21], it is highlighted that previous works rarely consider that the real-time location information of the mobile destination might be unavailable due to sparse network density. With this in mind, approach-androam (AaR) [7] adopts historical geographic information including location, moving speed recorded in the past to estimate a movement range of destination, and replicates messages using a two-phase routing scheme. Converge-and-diverge (CaD) [8] is further proposed to target the low routing overhead, while maintaining the delivery latency within an acceptable level.

\section{B. Spray-Based Routing Schemes}

The schemes in this branch limit the replication redundancy by an initialized defined copy ticket value $L$, where $L$ implies that only $(L-1)$ copies of a message can be replicated in the network. Here, the binary version of spray-and-wait (SaW) [12] has been proven effective in fast distributing $(L-1)$ message copies, by using a binary-tree-based distribution mechanism.

1) Based on Historical Encounter Information: Previous works [13], [22] further spray message copies to a better qualified candidate node based on the utility metric. Borrowing from the utility metric adopted in [23], spray-and-focus $(\mathrm{SaF})$ [12] adopts the focus phase instead of the wait phase, decreasing the delivery delay via a utility forwarding approach. Here, the focus phase relies on forwarding message copies in a multihop way via the last encounter time. This is different from binary SaW in which the message with one remaining copy ticket is only relayed to its destination. Furthermore, region-based [24] takes into account the region concept, enabling message forwarding within a region and message spraying between regions.

2) Based on Geographic Information: GeoSpray [14] borrows the geometric metric of GeOpps [25] for candidate node selection, requiring additional map topology information to find the nearest point (NP) via the navigation system. However, this scheme only considers that the destination is stationary and highly relies on the selection of NP via a map topology, particularly without handling the local maximum problem.

\section{Key Contributions}

Here, a summary of the related work is shown in Fig. 1. To the best of our knowledge, GSaR is the first geographic scheme in the literature, by using historical geographic information of the mobile destination, handling the local maximum under the design of a spray-based routing methodology. Different from our previous works [7], [8], which are characterized as utility-replication-based routing schemes, GSaR is advanced as follows.

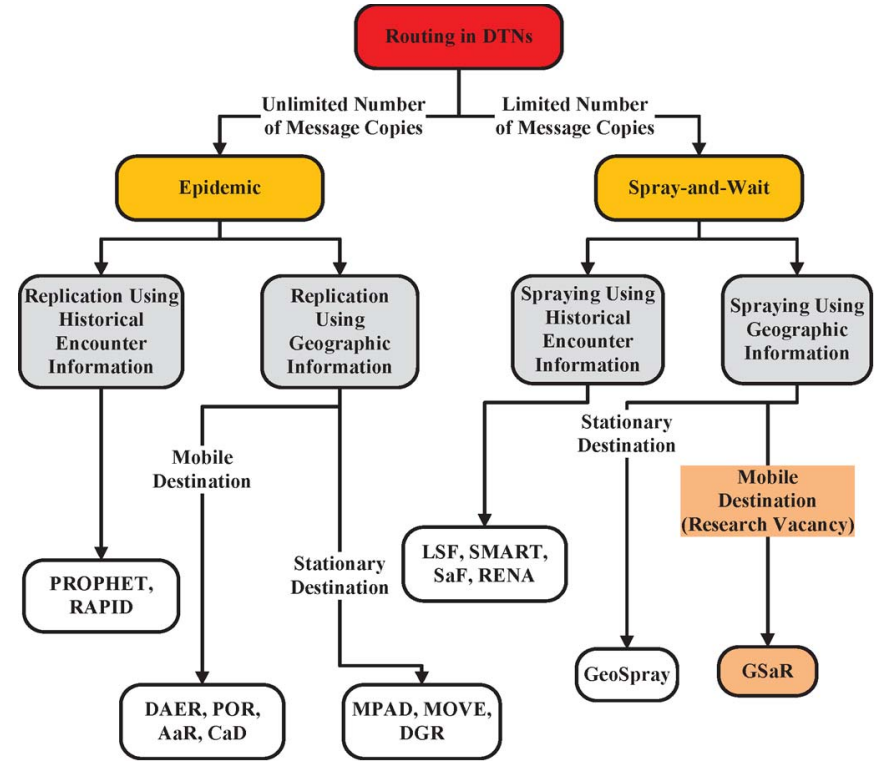

Fig. 1. Overview of Related Work.

- GSaR adopts the average historical moving speed to estimate the movement range of the destination. Given the nature of spraying messages and distributing message copy tickets, GSaR further considers the movement status when the encountered node is moving away from the movement range estimated for the destination.

- Upon this characteristic, the investigation of DF is considered in the design of GSaR, mainly for overcoming the limitation of a routing decision, enhancing for handling the local maximum problem, and reducing routing overhead.

- GSaR is with the design to make a routing decision if the historical geographic information of the destination is unavailable. Therefore, it is entitled with an enhanced message management framework for transmission and deletion, based on the awareness of destination.

\section{ASSUMption AND OVERVIEW}

We assume that each node is equipped with the Global Positioning System (GPS) to obtain its own real-time geographic information, including moving direction, current location, and moving speed, where the factor of GPS error is not taken into account in this paper. When pairwise nodes encounter, they will exchange, record, and update the historical geographic information of each other. Here, a slotted-based collision-avoidance MAC protocol is applied, in which only one connection for each message transmission is set up at each time slot.

Considering the sparse network density in DTNs, we rely solely on the basic ability of a node to communicate within its one-hop neighbor node; thus, the interference from a large transmission range is not taken into account. When pairwise nodes encounter, the routing decision is made based on whether the encountered node has better potential for message delivery. Otherwise, the message is carried until the destination is in proximity. The target of a routing scheme in DTNs is to achieve high delivery ratio with low routing overhead, whereas the 
TABLE I

LIST OF NOTATIONS

\begin{tabular}{|c|c|c|c|}
\hline$N_{i}$ & Message carrier & $D_{i, d}$ & Distance from the location of $N_{i}$ to $L(d)_{i, j}$, similarly for $D_{j, d}$ \\
\hline$N_{j}$ & Encountered node & $\theta_{i}$ & Moving direction of $N_{i}$, similarly for $\theta_{j}$ \\
\hline$N_{d}$ & Message destination & $\omega_{i, j}$ & Encounter angle between $N_{i}$ and $N_{j}$ \\
\hline$M$ & Message carried by $N_{i}$ & $\phi_{i, d}$ & Relative angle between $\theta_{i}$ and $D_{i, d}$, similarly for $\phi_{j, d}$ \\
\hline$T_{M}^{i n i}$ & Initialized message lifetime & $T_{i, d}^{t o}$ & $\begin{array}{l}\text { Time duration for } N_{i} \text { moving towards the movement range estimated } \\
\text { for } N_{d} \text {, similarly for } T_{j, d}^{t o}\end{array}$ \\
\hline$T_{M}^{e l a}$ & Elapsed time since message generation & $D_{i, d}^{a w}$ & $\begin{array}{l}\text { Distance for } N_{i} \text { moving away from movement range estimated for } \\
N_{d} \text {, similarly for } D_{j, d}^{a w}\end{array}$ \\
\hline$t_{i, d}$ & $\begin{array}{l}\text { Historical encounter time between } N_{i} \text { and } N_{d} \text { recorded by } N_{i} \text {, } \\
\text { similarly for } t_{j, d}\end{array}$ & $T_{i, d}^{i n}$ & $\begin{array}{l}\text { Time duration for } N_{i} \text { moving within the movement range estimated } \\
\text { for } N_{d} \text {, similarly for } T_{j, d}^{i n}\end{array}$ \\
\hline$L\left(t_{i, d}\right)$ & $\begin{array}{l}\text { Historical location }\left(x_{d}, y_{d}\right) \text { of } N_{d} \text { recorded by } N_{i} \text { at } t_{i, d}, \text { similarly } \\
\text { for } L\left(t_{j, d}\right)\end{array}$ & $V_{M}^{t o}$ & Threshold value for recording $T_{j, d}^{t o}$ \\
\hline$\Psi\left(t_{i, d}\right)$ & $\begin{array}{l}\text { Historical geographic information of } N_{d} \text { recorded by } N_{i} \text { at } t_{i, d} \text {, } \\
\text { similarly for } \Psi\left(t_{j, d}\right)\end{array}$ & $V_{M}^{a w}$ & Threshold value for recording $D_{j, d}^{a w}$ \\
\hline$t(d)_{i, j}$ & $\begin{array}{l}\text { Recent historical encounter time between } N_{d} \text { obtained from } t_{i, d} \text { and } \\
t_{j, d}\end{array}$ & $V_{M}^{i n}$ & Threshold value for recording $T_{j, d}^{i n}$ \\
\hline$L(d)_{i, j}$ & Recent historical location of $N_{d}$ obtained from $\Psi\left(t_{i, d}\right)$ and $\Psi\left(t_{j, d}\right)$ & $W$ & Time window for calculating $D_{i, d}^{a w}$ and $D_{j, d}^{a w}$ \\
\hline$K$ & Total number of nodes in the network & $C_{M}$ & Copy ticket for $M$ \\
\hline$t_{\text {cur }}$ & Current time in the network & $L$ & Initialized value for $C_{M}$ \\
\hline$S_{d}^{a v g}$ & Recorded historically average moving speed of $N_{d}$ & $P_{M}$ & Message priority \\
\hline$R_{d}$ & Radius of the movement range estimated for $N_{d}$ & $\operatorname{Bin}_{(\text {high })}$ & High priority buffer space \\
\hline$S_{i}$ & Moving speed of $N_{i}$, similarly for $S_{j}$ & $\operatorname{Bin}_{(\text {low })}$ & Low priority buffer space \\
\hline
\end{tabular}

delivery delay is considered to be the least important due to the delay-tolerant nature of applications in DTNs.

The design of GSaR consists of the following functions.

Information Update: When pairwise nodes encounter, their current location, encounter time, and moving speed are exchanged by each other. This information is recorded in a local routing table, to estimate the movement range of each node. In addition to this, if both of these two nodes have knowledge of a common node encountered in the past, the information in relation to that node is then updated toward the value recorded at a more recent time.

Spray Phase: Given the updated information, a movement range for the destination is estimated, to support the following three cases for the message spraying process. Here, each message records a copy ticket value $C_{M}$, where $\left(C_{M} \leq L\right)$ implies how many times it can be replicated in the future. In this paper, GSaR mainly adopts the binary spray process to fast distribute the $(L-1)$ message copies. This means that the replicated message is entitled with $C_{M} / 2$ copy tickets, whereas the original message maintains the rest $\left(C_{M}-\left(C_{M} / 2\right)\right)$ copy tickets.

- Based on selecting the candidate node moving toward this target range fast, the equal distribution of message copy tickets enables more message copies to be sprayed within this target range, which reduces delivery delay.

- Moreover, a long duration for a mobile node moving within this range contributes to message delivery by either encountering the destination directly or keep on spraying the residual number of message copies.

- In contrast, when moving away from this range, the node that is close to this range measured within a time window is selected as the candidate node, with only $\left(C_{M}=1\right)$ copy tickets distributed, to reduce the redundancy for spraying message copies.

In the worst case that the movement range estimated for the destination cannot be estimated due to infrequent encounter opportunity, as information in relation to the destination is unavailable, messages are sprayed considering the encounter angle and moving speed of pairwise nodes. Here, a larger encounter angle implies that two nodes are moving away from each other with a different relative direction, which contributes to an effective spraying process. Given that pairwise encountered nodes are moving in a consistent direction, spraying messages to that with faster moving speed expedites the spray process.

Relay Phase: When each message has been fully sprayed until $\left(C_{M}=1\right)$, these message copies are then forwarded using a single copy, following independent $(L-1)$ paths. Here, the selection of the candidate node is based on the historical record, i.e., about the best potential to move toward the movement range estimated for the destination, rather than selecting that with a better potential. The motivation behind this is to further reduce routing overhead, by filtering the node that does not significantly contribute to message delivery.

Message Management: Due to the intermittent connectivity in DTNs, messages may not be successfully transmitted. Moreover, given the nature of SCF routing behavior, messages are stored in the nodal buffer space for a long time. Considering that the encounter duration between two nodes is limited, the order for message transmission plays an important role in the routing performance. Considering the limited buffer space, it is essential to delete the least important message to allocate the buffer space for an incoming message. Since GSaR only generates a limited number of copies of a message in the network, if one copy is successfully delivered, a method to delete other copies of this message in a timely way is also essential to release buffer space for those undelivered messages.

\section{Detailed Design}

With the introduction of the given functions, we detail the design of GSaR in the following sections via the important notations listed in Table I. 
TABLE II

Historical Geographic Information $\Psi\left(t_{1,5}\right)$ FOR $N_{5}$, BASED ON THE VIEW OF $N_{1}$

\begin{tabular}{|c|c|c|c|}
\hline \multicolumn{4}{|c|}{ Routing Table of $N_{1}$} \\
\hline \hline ID of the Historically Encountered Node & Historical Location & Historically Average Moving Speed & Historical Encounter Time \\
\hline$N_{5}$ & $L\left(t_{1,5}\right)=(1000,2000)$ & $S_{5}^{\text {avg }}=6 \mathrm{~m} / \mathrm{s}$ & $t_{1,5}=1000 \mathrm{~s}$ \\
\hline
\end{tabular}

\section{A. Information Update}

We denote $N_{i}$ as the node that carries message $M$, whereas $N_{j}$ is the encountered node without carrying this message. Here, $N_{d}$ is as the destination node of $M$.

Based on the view of $N_{i}$, the required historical geographic information $\Psi\left(t_{i, d}\right)$ for $N_{d}$ contains the following records.

- $t_{i, d}$ : historical encounter time between $N_{i}$ and $N_{d}$, recorded by $N_{i}$;

- $L\left(t_{i, d}\right)$ : historical location $\left(x_{d}, y_{d}\right)$ of $N_{d}$, recorded by $N_{i}$ at $t_{i, d}$;

- $S_{d}^{\text {avg }}$ : historical average moving speed of $N_{d}$, as recorded by $N_{i}$. Note that $S_{d}^{\text {avg }}$ is an average value of the cumulative moving speed of $N_{d}$ at each encounter time.

As an example illustrated in Table II, $\Psi\left(t_{1,5}\right)$ is denoted as the historical geographic information for $N_{5}$, consisting of the historical location $L\left(t_{1,5}\right)=(1000,2000)$ and average moving speed $S_{5}^{\text {avg }}=6 \mathrm{~m} / \mathrm{s}$ recorded in the past, when $N_{1}$ encountered $N_{5}$ at $t_{1,5}=1000 \mathrm{~s}$. Given that $N_{1}$ encounters another node $N_{2}$, with $\Psi\left(t_{2,5}\right)$, where $t_{2,5}=2200 \mathrm{~s}$, then the historical information about $N_{5}$, as recorded in $\Psi\left(t_{1,5}\right)$, is updated according to that of $\Psi\left(t_{2,5}\right)$ because of $\left(t_{2,5}>t_{1,5}\right)$. In another case, that if $N_{2}$ did not meet $N_{5}$ in the past, then $\Psi\left(t_{2,5}\right)$ is simply updated toward that of $\Psi\left(t_{1,5}\right)$. The example described herein can be referred to the presentation between lines 5 and 13 in Algorithm 1.

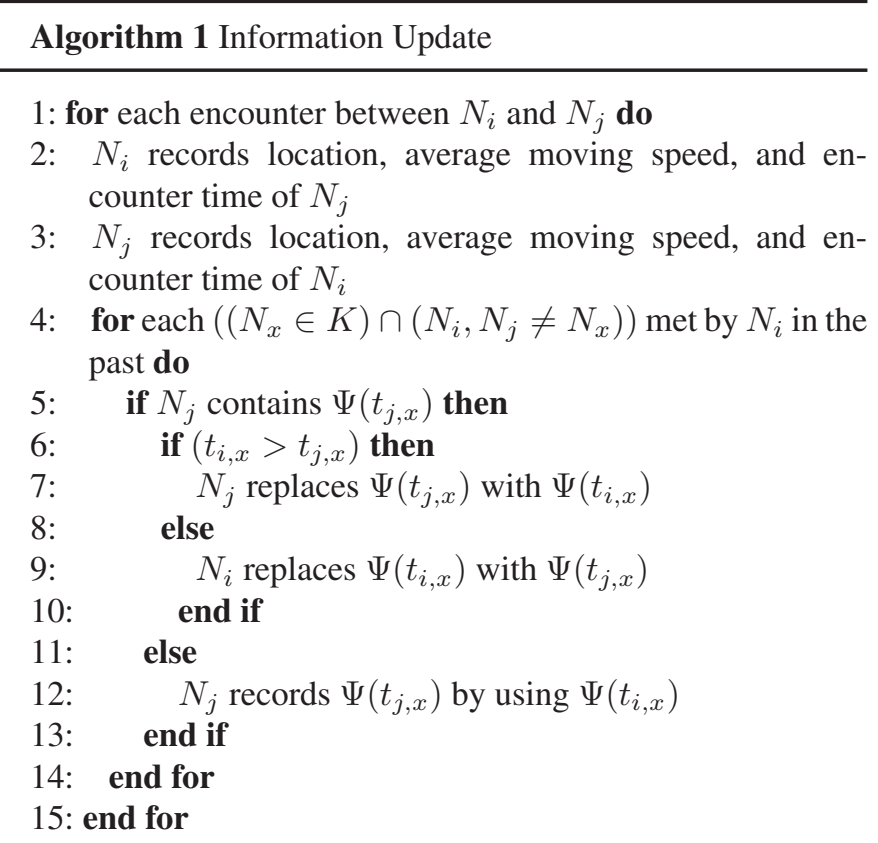

\section{B. Candidate Node Selecting}

We denote $L(d)_{i, j}$ as the recent historical location of $N_{d}$ obtained from $\Psi\left(t_{i, d}\right)$ and $\Psi\left(t_{j, d}\right)$, following the given example about information update. Depending on the current time in the network $t_{\text {cur }}$, the estimated movement range for $N_{d}$ is assumed as a circle, where its radius $R_{d}$ is calculated as

$$
R_{d}=S_{d}^{\mathrm{avg}} \times\left[t_{\mathrm{cur}}-t(d)_{i, j}\right] .
$$

Here, $t(d)_{i, j}=\max \left[t_{i, d}, t_{j, d}\right]$ is denoted as a more recent time value between $t_{i, d}$ and $t_{j, d}$. Based on the distance $D_{j, d}$ measured from $N_{j}$ to $L(d)_{i, j}$ and the moving direction of $N_{j}$ as denoted by $\phi_{j, d}$, the following cases are considered for selecting the candidate node.

1) The $\left(\left(D_{j, d} \geq R_{d}\right) \cap\left(\phi_{j, d}<(\pi / 2)\right)\right)$ Case: As illustrated in Fig. 2(a), this case happens when $N_{j}$ is moving toward the movement range estimated for $N_{d}$. We qualify $N_{j}$ via its time duration $T_{j, d}^{\text {to }}$ to move toward the movement range estimated for $N_{d}$, where

$$
T_{j, d}^{\mathrm{to}}=\frac{D_{j, d}-R_{d}}{\cos \phi_{j, d} \times S_{j}} .
$$

Given $\left(T_{i, d}^{\mathrm{to}}>T_{j, d}^{\mathrm{to}}\right), N_{j}$ is selected as a better relay node due to a faster proximity to this range, for reducing the delivery delay.

To calculate $T_{i, d}^{\text {to }}$ requires conditions $\left(\left(D_{i, d}>R_{d}\right) \cap\left(\phi_{i, d}<\right.\right.$ $(\pi / 2)))$. However, $T_{i, d}^{\text {to }}$ is invalid either if $\left(D_{i, d}<R_{d}\right)$ or $\left(\phi_{i, d} \geq(\pi / 2)\right)$, because the negative value of $T_{i, d}^{\text {to }}$ is unable for comparison. Note that in the special case where $\left(T_{i, d}^{\text {to }}=0\right)$, the routing decision for message $M$ is omitted. Therefore, by using DF to overcome this limitation, a threshold value $V_{M}^{\text {to }}$ is cached for message $M$, and we convert conditions

$$
\left(\left(T_{i, d}^{\mathrm{to}}>T_{j, d}^{\mathrm{to}}\right) \cap\left(D_{i, d}>R_{d}\right) \cap\left(\phi_{i, d}<\frac{\pi}{2}\right)\right)
$$

into

$$
\left(V_{M}^{\mathrm{to}}>T_{j, d}^{\mathrm{to}}\right)
$$

Here, $V_{M}^{\text {to }}$ is denoted as an updated value of $T_{j, d}^{\text {to }}$. Thereby, when conditions $\left(\left(D_{j, d} \geq R_{d}\right) \cap\left(\phi_{j, d}<(\pi / 2)\right) \cap\right.$ $\left.\left(V_{M}^{\mathrm{to}}>T_{j, d}^{\mathrm{to}}\right)\right)$ are satisfied, the value of $T_{j, d}^{\mathrm{to}}$ is recorded into $V_{M}^{\text {to }}$. In GSaR, each node will locally check its individual motion status every $1 \mathrm{~s}$, to calculate its individual moving speed and direction. During communication, this status information will be exchanged between two encountered nodes, such that they can obtain the speed and direction of each other.

Different from the original application of DF, $V_{M}^{\text {to }}$ is initialized with an infinitely large value, which considers the situation that $\left(D_{i, d}<R_{d}\right)$ or $\left(\phi_{i, d} \geq(\pi / 2)\right)$ when $M$ is generated by $N_{i}$. Furthermore, initializing $\left(V_{M}^{\text {to }}=+\infty\right)$ avoids failure of a 


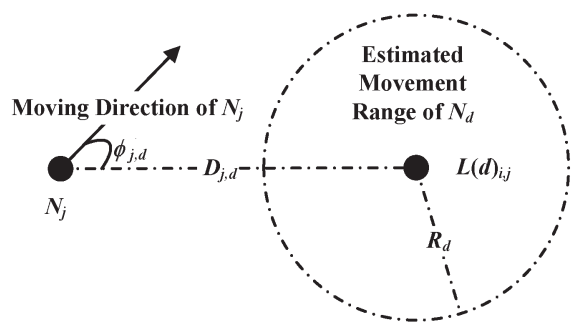

(a)

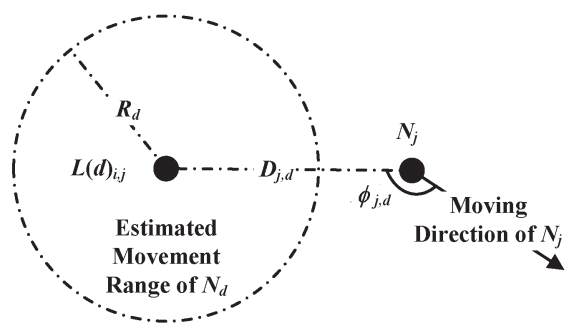

(b)

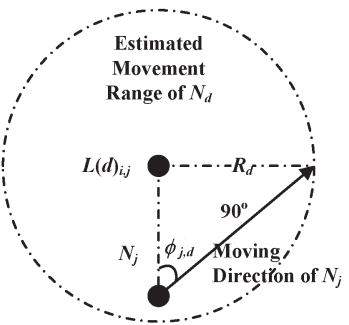

(c)

Fig. 2. Three cases for selecting candidate node. (a) The $\left(\left(D_{j, d} \geq R_{d}\right) \cap\left(\phi_{j, d}<(\pi / 2)\right)\right)$ case. (b) The $\left(\left(D_{j, d} \geq R_{d}\right) \cap\left(\phi_{j, d} \geq(\pi / 2)\right)\right)$ case. (c) The $\left(D_{j, d}<R_{d}\right)$ case.

routing decision, particularly if $\left(D_{i, d}=R_{d}\right)$ happens at message generation. In light of this, the stated motivation focuses on comparing this time-based utility metric, between the currently encountered node and previously encountered node, instead of comparing that between the message carrier and currently encountered node. Meanwhile, if $N_{j}$ already has a copy of $M$, it is essential to update $V_{M}^{\text {to }}$ toward a smaller value between these two messages carried by both $N_{i}$ and $N_{j}$.

Although using the time duration measured along the progress distance to the destination may also select the node whose $\phi_{j, d}$ is close to $\pi / 2$, using DF will make the routing decision an optimality. This is because apart from the gradually decreased $^{4}\left(D_{j, d}-R_{d}\right)$, the gradual update for $V_{M}^{\text {to }}$ implies either the smallest $\phi_{j, d}$ or largest $S_{j}$. As such, the quality of the selected candidate node will be gradually optimized and converged. In contrast, when using $\left(\left(T_{i, d}^{\mathrm{to}}>T_{j, d}^{\mathrm{to}}\right) \cap\left(D_{i, d}>\right.\right.$ $\left.\left.R_{d}\right) \cap\left(\phi_{i, d}<(\pi / 2)\right)\right)$ for comparison, $N_{j}$ might be selected to carry $M$, given that $\phi_{j, d}$ is close to $\pi / 2$ while $S_{j}$ is large. Consequently, although $M$ has been relayed for several hops, it may not be sprayed close to the movement range estimated for $N_{d}$.

2) The $\left(\left(D_{j, d} \geq R_{d}\right) \cap\left(\phi_{j, d} \geq(\pi / 2)\right)\right)$ Case: As shown in Fig. 2(b), this case happens when $N_{j}$ is currently moving away from the movement range estimated for $N_{d}$. We qualify $N_{j}$ based on its projected distance $D_{j, d}^{\text {aw }}$ as calculated in (5), which is estimated from $N_{j}^{\prime}$ to the edge of the movement range estimated for $N_{d}$, i.e.,

$$
D_{j, d}^{\mathrm{aw}}=D_{j, d}-W \times \cos \phi_{j, d} \times S_{j}-R_{d} .
$$

Here, $N_{j}^{\prime}$ is denoted as the expected location estimated within a time window $W$. Upon this, the condition $\left(D_{i, d}^{\text {aw }}>D_{j, d}^{\text {aw }}\right)$ implies that $N_{j}$ is closer to the movement range estimated for $N_{d}$.

However, we further note that calculating $D_{i, d}^{\text {aw }}$ requires $\left(\left(D_{i, d}>R_{d}\right) \cap\left(\phi_{i, d} \geq(\pi / 2)\right)\right)$, which limits the routing decision either if $\left(D_{i, d}<R_{d}\right)$ or $\left(\phi_{i, d}<(\pi / 2)\right)$. This is because the intention in this case considers that both $N_{i}$ and $N_{j}$ are moving away from the movement range related to $N_{d}$, and only let that with a closer proximity to this range keep carrying

${ }^{4}$ Considering that the information for $N_{d}$ has not been updated via Algorithm $1, D_{j, d}$ is always decreased, given $\left(\phi_{j, d}<(\pi / 2)\right)$. Meanwhile, $R_{d}$ is increased due to rare encounter. messages. Similar to the previous investigation on DF, another threshold value $V_{M}^{\text {aw }}$ is cached in message, then conditions

$$
\left(\left(D_{i, d}^{\mathrm{aw}}>D_{j, d}^{\mathrm{aw}}\right) \cap\left(D_{i, d}>R_{d}\right) \cap\left(\phi_{i, d} \geq \frac{\pi}{2}\right)\right)
$$

are converted into

$$
\left(V_{M}^{\mathrm{aw}}>D_{j, d}^{\mathrm{aw}}\right)
$$

By referring to the initialization of $V_{M}^{\text {to }}, V_{M}^{\text {aw }}$ is also set with an infinitely large value and gradually updated toward a smaller value.

Due to updating $V_{M}^{\text {aw }}$, the candidate node will be gradually selected as that either with the $\phi_{j, d}$ close to $\pi / 2$ or with the smallest $S_{j}$. Thus, the value of $\left(W \times \cos \phi_{j, d} \times S_{j}\right)$ is quite small, considering the sparse network density and highly dynamic movement. In particular, even assuming $\left(\phi_{j, d}=(\pi / 2)\right)$, the candidate node that is closer to the movement range estimated for $N_{d}$ will be selected in the future, given that $V_{M}^{\text {aw }}$ has been updated to $\left(D_{j, d}-0-R_{d}\right)$.

3) The $\left(D_{j, d}<R_{d}\right)$ Case: Considering that $N_{j}$ has been within the movement range estimated for $N_{d}$, as shown in Fig. 2(c), we define the time duration $T_{j, d}^{\text {in }}$ for $N_{j}$ to move within this range, where

$$
T_{j, d}^{\mathrm{in}}=\frac{\sqrt{\left(R_{d}\right)^{2}-\left(D_{j, d} \times \sin \phi_{j, d}\right)^{2}}+D_{j, d} \times \cos \phi_{j, d}}{S_{j}} .
$$

The detail of calculating $T_{j, d}^{\text {in }}$ can be referred to [7]. Here, $N_{j}$ is selected as the candidate node given $\left(T_{j, d}^{\mathrm{in}}>T_{i, d}^{\mathrm{in}}\right)$, as a longer time duration moving within the movement range estimated for $N_{d}$ implies a higher possibility to encounter $N_{d}$ directly.

We note that calculating $T_{i, d}^{\text {in }}$ also requires that $N_{i}$ is within the target range, as given by $\left(D_{i, d}<R_{d}\right)$. Following the previous discussion on using DF, here, conditions

$$
\left(\left(T_{i, d}^{\mathrm{in}}<T_{j, d}^{\mathrm{in}}\right) \cap\left(D_{i, d}<R_{d}\right)\right)
$$

are converted into

$$
\left(V_{M}^{\mathrm{in}}<T_{j, d}^{\mathrm{in}}\right)
$$

Note that $V_{M}^{\mathrm{in}}$ is the corresponding threshold value defined in this case, to record the value of $T_{j, d}^{\text {in }}$. Different from the initialization of $V_{M}^{\text {to }}$ and $V_{M}^{\text {aw }}, V_{M}^{\text {in }}$ is set to 0 and gradually updated toward a larger value. Therefore, by obtaining the 

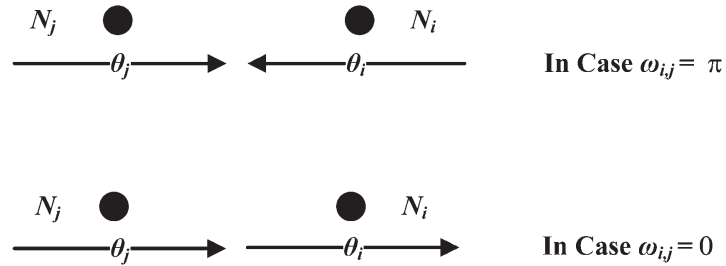

Fig. 3. Encounter given different $\omega_{i, j}$.

largest value of $V_{M}^{\text {in }}$ recorded in the past, message copies are ideally carried by, at most, $(L-1)$ candidate nodes within this range.

\section{Spray Phase}

Following the overview of GSaR presented in Section III, the specific routing scheme is detailed here.

1) When $L(d)_{i, j}$ is Unavailable: If the historical geographic information is unavailable, messages are only sprayed based on the local movement status of pairwise encountered nodes. Here, the encounter angle $\omega_{i, j}$ is calculated as

$$
\omega_{i, j}= \begin{cases}\left|\theta_{i}-\theta_{j}\right|, & \text { if }\left(\left|\theta_{i}-\theta_{j}\right| \leq \pi\right) \\ 2 \pi-\left|\theta_{i}-\theta_{j}\right|, & \text { else. }\end{cases}
$$

Considering $\left(\omega_{i, j}>(\pi / 2)\right)$ shown in Fig. 3, we propose to spray messages with $C_{M} / 2$ copy tickets to $N_{j}$. This is because if pairwise nodes are moving in a consistent direction, spraying messages does not significantly contribute to delivery. In contrast, if they are moving in different directions, spraying messages thus contributes to effective delivery, as the node holding the sprayed message copies may reach its destination on the move. This operation is presented between lines 11 and 13 in Algorithm 2.

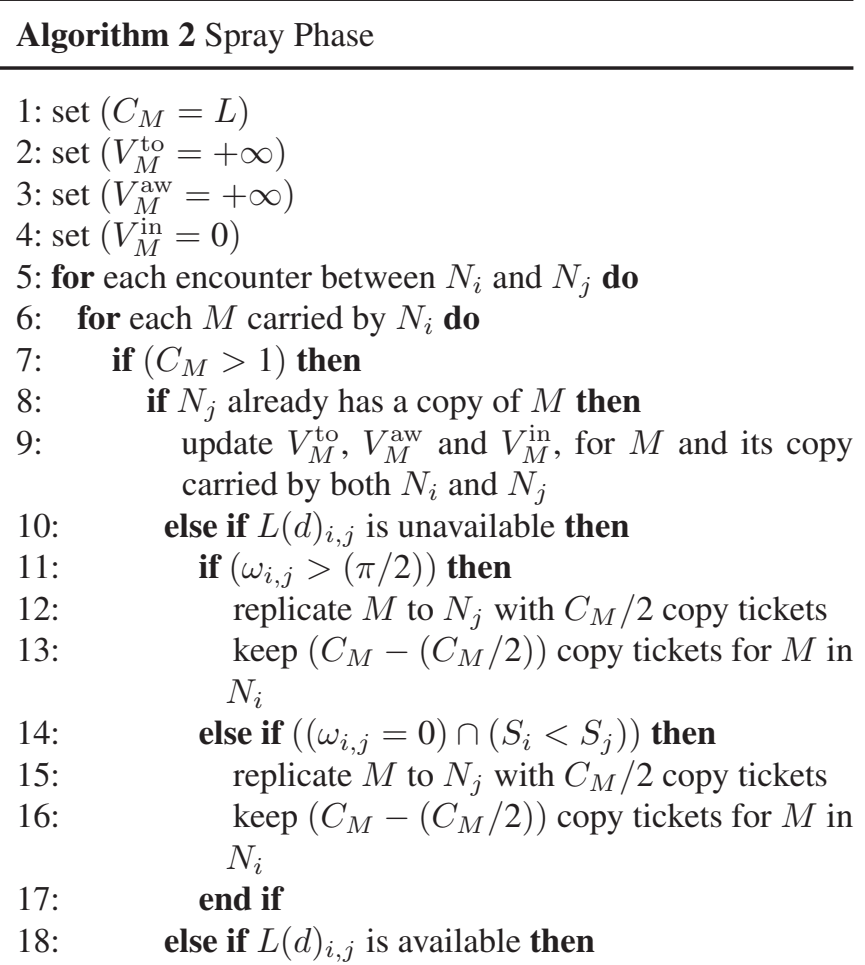

19:

20

21:

22

24:

25:

27:

28:

29

30 :

$31:$

32 :

33 :

$34:$

35

36:

37:

38:

39:

40:

\section{1: end for}

$$
\begin{aligned}
& \text { if } \quad\left(( D _ { j , d } \geq R _ { d } ) \cap ( \phi _ { j , d } < ( \pi / 2 ) ) \cap \left(V_{M}^{\text {to }}>\right.\right. \\
& \left.\left.T_{j, d}^{\text {to }}\right)\right) \text { then } \\
& \quad \text { update } V_{M}^{\text {to }} \text { toward } T_{j, d}^{\text {to }}
\end{aligned}
$$$$
\text { replicate } M \text { to } N_{j} \text { with } C_{M} / 2 \text { copy tickets }
$$
keep $\left(C_{M}-\left(C_{M} / 2\right)\right)$ copy tickets for $M$ in $N_{i}$

else $\quad$ if $\quad\left(\left(D_{j, d} \geq R_{d}\right) \cap\left(\phi_{j, d}<(\pi / 2)\right) \cap\right.$ $\left.\left(V_{M}^{\text {to }}>T_{M}^{\text {ini }}-T_{M}^{\text {ela }}\right)\right)$ then replicate $M$ to $N_{j}$ with $C_{M} / 2$ copy tickets keep $\left(C_{M}-\left(C_{M} / 2\right)\right)$ copy tickets for $M$ in $N_{i}$

else $\quad$ if $\quad\left(\left(D_{j, d} \geq R_{d}\right) \cap\left(\phi_{j, d} \geq(\pi / 2)\right) \cap\right.$ $\left.\left(V_{M}^{\mathrm{aw}}>D_{j, d}^{\mathrm{aw}}\right)\right)$ then update $V_{M}^{\text {aw }}$ toward $D_{j, d}^{\text {aw }}$

replicate $M$ to $N_{j}$ with $\left(C_{M}=1\right)$ copy ticket

keep $\left(C_{M}-1\right)$ copy tickets for $M$ in $N_{i}$ else if $\left(\left(D_{j, d}<R_{d}\right) \cap\left(V_{M}^{\text {in }}<T_{j, d}^{\text {in }}\right)\right)$ then update $V_{M}^{\text {in }}$ toward $T_{j, d}^{\text {in }}$ replicate $M$ to $N_{j}$ with $C_{M} / 2$ copy tickets keep $\left(C_{M}-\left(C_{M} / 2\right)\right)$ copy tickets for $M$ in $N_{i}$

else if $\left(\left(D_{j, d}<R_{d}\right) \cap\left(V_{M}^{\mathrm{in}}>T_{M}^{\mathrm{ini}}-T_{M}^{\mathrm{ela}}\right)\right)$ then

replicate $M$ to $N_{j}$ with $C_{M} / 2$ copy tickets keep $\left(C_{M}-\left(C_{M} / 2\right)\right)$ copy tickets for $M$ in $N_{i}$

$$
\text { end if }
$$

end if

end if

end for

As a special case where the encounter angle is $\left(\omega_{i, j}=0\right), N_{j}$ is selected to spray messages if its moving speed $S_{j}$ is faster than $S_{i}$. Following the presentation between lines 14 and 16 in Algorithm 2, this is because a faster node would contribute to fast delivery, particularly considering that messages are rarely sprayed, even with short lifetime.

2) When $L(d)_{i, j}$ is Available: In this case, the routing decision utilizes the threshold values $V_{M}^{\text {to }}, V_{M}^{\text {aw }}$, and $V_{M}^{\text {in }}$ defined in message header, as shown in Fig. 4. Then, the following operations are made, based on the selected candidate node as discussed in Section IV-B.

- Referring to Fig. 2(a) that $\left(\left(D_{j, d} \geq R_{d}\right) \cap\left(\phi_{j, d}<\right.\right.$ $\left.(\pi / 2)) \cap\left(V_{M}^{\text {to }}>T_{j, d}^{\text {to }}\right)\right), N_{i}$ distributes $C_{M} / 2$ copy tickets for the message replicated to $N_{j}$. Following lines 19 and 22 in Algorithm 2, the motivation behind this is to fast spray message copies toward the movement range estimated for $N_{d}$, as the delivery ratio is increased by enabling more message copies to exist within this range.

- If considering the local maximum problem that $\left(\left(D_{j, d} \geq\right.\right.$ $\left.\left.R_{d}\right) \cap\left(\phi_{j, d}<(\pi / 2)\right) \cap\left(V_{M}^{\mathrm{to}} \leq T_{j, d}^{\mathrm{to}}\right)\right), \quad M$ cannot be sprayed until a node holding $\left(V_{M}^{\text {to }}>T_{j, d}^{\text {to }}\right)$ is encountered. In the worst case, this problem would delay or even degrade message delivery, particularly if the message is 


\begin{tabular}{|c|c|c|c|c|c|c|c|}
\hline Destination & Source & $\mathrm{C}_{\mathrm{M}}$ & $\mathrm{V}^{\mathrm{t}}{ }_{\mathrm{M}}$ & $\mathrm{V}^{\text {aw }}{ }_{\mathrm{M}}$ & $\mathrm{V}^{\text {in }}{ }_{\mathrm{M}}$ & $\begin{array}{c}\text { Initialized } \\
\text { Lifetime }\end{array}$ & $\begin{array}{c}\text { Creation } \\
\text { Time }\end{array}$ \\
\hline
\end{tabular}

Fig. 4. Message structure of GSaR.

close to its expiration deadline. With this in mind, we have the following inequality:

$$
\left(T_{M}^{\mathrm{ela}}+T_{i, d}^{\mathrm{to}} \leq T_{M}^{\mathrm{ini}}\right) .
$$

Here, $T_{M}^{\mathrm{ela}}$ is the elapsed time starting from message generation, and $T_{M}^{\mathrm{ini}}$ is the initialized lifetime of the message. Considering that $\left(T_{M}^{\mathrm{ini}}-T_{M}^{\mathrm{ela}}\right)$ is calculated as the remaining message lifetime, conditions $\left(\left(T_{i, d}^{\mathrm{to}}>T_{M}^{\mathrm{ini}}-T_{M}^{\mathrm{ela}}\right) \cap\right.$ $\left.\left(D_{i, d}>R_{d}\right) \cap\left(\phi_{i, d}<(\pi / 2)\right)\right)$ imply that $M$ would expire before $T_{i, d}^{\text {to }}$. To this end, $M$ is sprayed to $N_{j}$ with $C_{M} / 2$ copy tickets for handling this local maximum problem, aiming that $N_{j}$ would encounter other nodes with a smaller value than $T_{i, d}^{\text {to }}$ at upcoming encounter opportunity.

Recall that calculating $T_{i, d}^{\text {to }}$ is inherently with limitations, either if $\left(D_{i, d}<R_{d}\right)$ or $\left(\phi_{i, d} \geq(\pi / 2)\right)$, we then convert the inequality

$$
\left(\left(T_{i, d}^{\mathrm{to}}>T_{M}^{\mathrm{ini}}-T_{M}^{\mathrm{ela}}\right) \cap\left(D_{i, d}>R_{d}\right) \cap\left(\phi_{i, d}<\frac{\pi}{2}\right)\right)
$$

into

$$
\left(V_{M}^{\mathrm{to}}>T_{M}^{\mathrm{ini}}-T_{M}^{\mathrm{ela}}\right) .
$$

By removing conditions $\left(\left(D_{i, d}>R_{d}\right) \cap\left(\phi_{i, d}<(\pi / 2)\right)\right)$, inequality (14) implies that even $V_{M}^{\text {to }}$, as the smallest value of $T_{j, d}^{\mathrm{to}}$ recorded in the network, is still longer than the remaining message lifetime $\left(T_{M}^{\mathrm{ini}}-T_{M}^{\mathrm{ela}}\right)$. As highlighted between lines 23 and 25 in Algorithm 2, $V_{M}^{\text {to }}$ is not updated in this case because of $\left(V_{M}^{\mathrm{to}} \leq T_{j, d}^{\mathrm{to}}\right)$.

- When $N_{j}$ is moving away from the movement range estimated for $N_{d}$, as given by $\left(\left(D_{j, d} \geq R_{d}\right) \cap\left(\phi_{j, d} \geq\right.\right.$ $(\pi / 2)))$, the message is sprayed to $N_{j}$ satisfying $\left(V_{M}^{\text {aw }}>\right.$ $\left.D_{j, d}^{\text {aw }}\right)$, with $\left(C_{M}=1\right)$ copy ticket distributed. Referring to Fig. 2(b), this is because it is beneficial to spray more message copies toward the movement range in relation to $N_{d}$ for reducing the delivery delay, rather than spraying them away from this range. Meanwhile, spraying the message with $\left(C_{M}=1\right)$ distributed copy ticket also increases the diffusion speed, where it will be performed by the relay phase introduced later on. This operation is presented between lines 26 and 29 in Algorithm 2.

- Considering that $\left(D_{j, d}<R_{d}\right)$ shown in Fig. 2(c), messages are sprayed to the candidate nodes that are with long time duration moving within the movement range estimated for the destination, this increases the possibility of directly encountering the destination. In light of this, as presented between lines 30 and $33, N_{i}$ replicates a message copy to $N_{j}$, with $C_{M} / 2$ copy tickets distributed given the condition $\left(V_{M}^{\mathrm{in}}<T_{j, d}^{\mathrm{in}}\right)$.

- Moreover, conditions $\left(\left(D_{j, d}<R_{d}\right) \cap\left(V_{M}^{\mathrm{in}}>T_{M}^{\mathrm{ini}}-\right.\right.$ $\left.\left.T_{M}^{\text {ela }}\right)\right)$ are used to continually spray message copies, if the local maximum problem $\left(\left(D_{j, d}<R_{d}\right) \cap\left(V_{M}^{\mathrm{in}} \geq T_{j, d}^{\mathrm{in}}\right)\right)$ happens. As highlighted between lines 34 and 36 in Algorithm 2, $V_{M}^{\text {in }}$ is not updated in this case because of $\left(V_{M}^{\mathrm{in}} \geq T_{j, d}^{\mathrm{in}}\right)$.

3) Property of Spray Phase: Here, we denote GSaR (WH) as the version without handling the local maximum problem in the spray phase. By referring to [26] that given a consistent message delivery ratio, direct delivery (DD) [27] achieves the upper bound delivery delay for any routing scheme in DTNs, in which the message is only relayed and delivered when the destination is in proximity.

In the worst case that if candidate nodes are unavailable, $\mathrm{GSaR}$ (WH) behaves as DD since messages are never relayed. By using $\left(\left(D_{j, d} \geq R_{d}\right) \cap\left(\phi_{j, d}<(\pi / 2)\right) \cap\left(V_{M}^{\text {to }}>\right.\right.$ $\left.\left.T_{M}^{\text {ini }}-T_{M}^{\text {ela }}\right)\right) \quad$ or $\left(\left(D_{j, d}<R_{d}\right) \cap\left(V_{M}^{\text {in }}>T_{M}^{\text {ini }}-T_{M}^{\text {ela }}\right)\right)$ to spray messages if better candidate nodes are unavailable, GSaR is less likely to behave as DD. Consequently, GSaR achieves a lower delivery delay than GSaR $(\mathrm{WH})$ by spraying $(L-1)$ message copies faster. Furthermore, since each of $(L-1)$ candidate nodes of a message is selected along a routing path independently in the relay phase, a longer delay in the spray phase adversely affects the delay of the message in the relay phase and, sequentially, deteriorates delivery ratio within the given expiration deadline.

\section{Relay Phase}

Algorithm 3 illustrates the detail of the relay phase. Given $\left(\left(D_{j, d} \geq R_{d}\right) \cap\left(\phi_{j, d}<(\pi / 2)\right) \cap\left(V_{M}^{\text {to }}>T_{j, d}^{\text {to }}\right)\right)$, as shown in Fig. 2(a), the message with $\left(C_{M}=1\right)$ copy ticket is forwarded to $N_{j}$ using a single copy only, toward the movement range estimated for $N_{d}$. Since this given message cannot be sprayed anymore, the relay phase further reduces the delay that message copies could move toward the movement range estimated for $N_{d}$, instead of awaiting the node that carries this message to reach the target range given its intrinsic mobility.

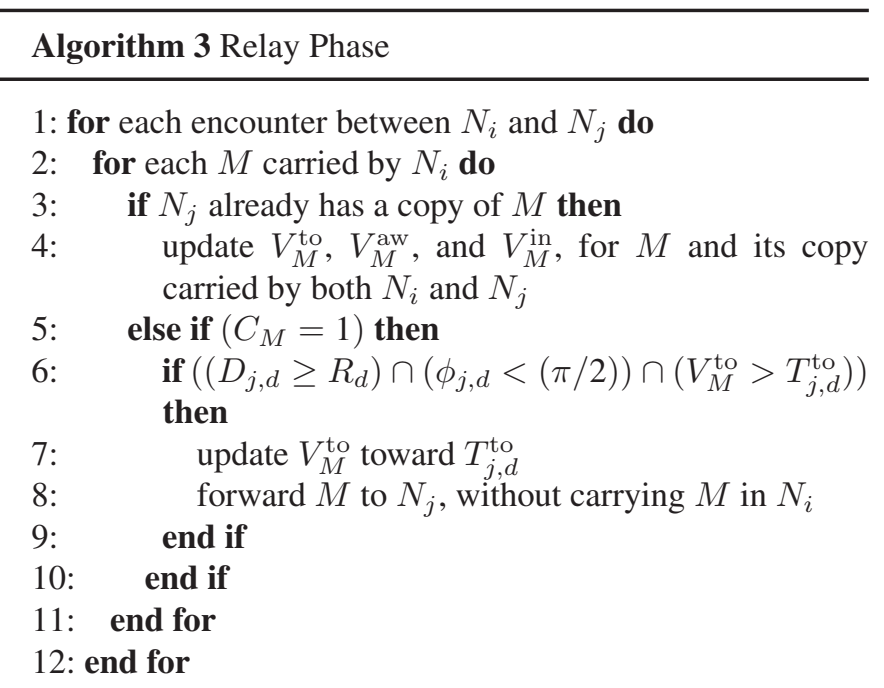

Considering $\left(\phi_{j, d} \geq(\pi / 2)\right)$, forwarding the message with $\left(C_{M}=1\right)$ copy ticket results in redundancy, because such operation does not contribute to relaying this given message toward the movement range in relation to $N_{d}$. Moreover, 
forwarding the message with the $\left(C_{M}=1\right)$ copy ticket within this range is also prevented. This is because the message has already been within its target range, and particularly, some of its copies may be carried by the candidate node moving within this range. Thus, the message delivery is achieved by relying on the mobility of $(L-1)$ candidate nodes.

Here, the routing decision will be converged, by comparing $V_{M}^{\text {to }}$ with $T_{j, d}^{\text {to }}$, where $V_{M}^{\text {to }}$ is the historical value updated between, at most, $(L-1)$ message copies in an ideal case. ${ }^{5}$ Since the message transmission is unidirectional, the message with $\left(C_{M}=1\right)$ copy ticket is always forwarded to the node with a lower value than $V_{M}^{\text {to }}$. Note that this operation is also similar to the design of achieving loop free in a traditional network, by setting a maximum value, such as hop count to prevent further message relay. Along with this, this given message is relayed to the node that holds the smallest value of $T_{j, d}^{\text {to }}$ recorded in the past, rather than being relayed to a node that only holds a smaller value than $T_{i, d}^{\mathrm{to}}$. As such, the number of transmissions for this copy is further reduced, in contrast to the local greedy nature that only comparing with the current $T_{i, d}^{\text {to }}$.

\section{E. Message Management}

1) Defining Message Priority: The priority $P_{M}$ to manage messages is defined as follows.

If both $N_{i}$ and $N_{j}$ do not obtain any information about destination $N_{d}, P_{M}$ is calculated as

$$
P_{M}=1-\left(1-\frac{\left(T_{M}^{\mathrm{ini}}-T_{M}^{\mathrm{ela}}\right)}{T_{M}^{\mathrm{ini}}}\right)^{C_{M}} .
$$

Based on the current copy ticket value $C_{M}$ and remaining message lifetime $\left(T_{M}^{\mathrm{ini}}-T_{M}^{\mathrm{ela}}\right)$, this equation considers the possibility of a message being delivered within its maximum expiration deadline $T_{M}^{\text {ini }}$. Therefore, both a larger value of $\left(T_{M}^{\mathrm{ini}}-T_{M}^{\mathrm{ela}}\right)$ and $C_{M}$ imply a higher delivery possibility. Given that $1-\left(\left(T_{M}^{\mathrm{ini}}-T_{M}^{\mathrm{ela}}\right) / T_{M}^{\mathrm{ini}}\right)^{C_{M}}$ is the probability that a message entitling with $C_{M}$ copy tickets is not delivered within the deadline, (15) thus presents the probability that at least one copy $^{6}$ of this message could be successfully delivered.

In another case, if the historical geographic information of $N_{d}$ is available, we classify $P_{M}$ depending on whether $N_{j}$ is currently within the estimated movement range for $N_{d}$.

- If $\left(D_{j, d} \geq R_{d}\right)$, the probability that $M$ reaches the movement range of $N_{d}$ before $\left(T_{M}^{\mathrm{ini}}-T_{M}^{\mathrm{ela}}\right)$ is calculated as $\left(\left(T_{M}^{\text {ini }}-T_{M}^{\text {ela }}\right)-V_{M}^{\text {to }}\right) /\left(T_{M}^{\text {ini }}-T_{M}^{\text {ela }}\right)$. Here, since $V_{M}^{\text {to }}$ will not be updated toward a smaller value in case that $\left(\left(\phi_{j, d} \geq(\pi / 2)\right) \cap\left(D_{j, d} \geq R_{d}\right)\right)$ during the routing process, a negative value of $\left(\left(T_{M}^{\text {ini }}-T_{M}^{\text {ela }}\right)-V_{M}^{\text {to }}\right)$ implies that $M$ will expire before being sprayed toward the target range.

\footnotetext{
${ }^{5}$ Although this message copy is not forwarded either given $\left(\left(D_{j, d} \geq R_{d}\right) \cap\right.$ $\left.\left(\phi_{j, d} \geq(\pi / 2)\right)\right)$ or $\left(D_{j, d}<R_{d}\right), V_{M}^{\text {aw }}$ and $V_{M}^{\text {in }}$ are still updated. This is because that other copies of this message may still be performed by the spray phase.

${ }^{6}$ It is referred to as the one with $\left(C_{M}=1\right)$ copy ticket only.
}

Next, by considering the copy ticket $C_{M}$, the message with a larger value of $C_{M}$ implies that it has not been extensively sprayed. Since a larger number of message copies will increase the message delivery probability, the message with a larger value of $C_{M}$ is more important. Then, we have

$$
P_{M}=1-\left(1-\frac{\left(T_{M}^{\mathrm{ini}}-T_{M}^{\mathrm{ela}}\right)-V_{M}^{\mathrm{to}}}{\left(T_{M}^{\mathrm{ini}}-T_{M}^{\mathrm{ela}}\right)}\right)^{C_{M}} .
$$

- Similarly, when considering $\left(D_{j, d}<R_{d}\right)$, a negative value of $\left(\left(T_{M}^{\mathrm{ini}}-T_{M}^{\mathrm{ela}}\right)-V_{M}^{\mathrm{in}}\right)$ implies that $M$ will expire, before the maximum time duration moving within the movement range estimated for $N_{d}$. In this case, we have

$$
P_{M}=1-\left(1-\frac{\left(T_{M}^{\mathrm{ini}}-T_{M}^{\mathrm{ela}}\right)-V_{M}^{\mathrm{in}}}{\left(T_{M}^{\mathrm{ini}}-T_{M}^{\mathrm{ela}}\right)}\right)^{C_{M}}
$$

In light of this, the message priority is defined by (18), where $P_{M}$ is calculated in an ideal case. ${ }^{7}$ Meanwhile, the definition of $P_{M}$ implies to transmit those messages processed by the spray phase with a higher priority, due to a larger value of $C_{M}$.

$$
P_{M}= \begin{cases}1-\left(1-\frac{\left(T_{M}^{\mathrm{ini}}-T_{M}^{\mathrm{ela}}\right)}{T_{M}^{\mathrm{ini}}}\right)^{C_{M}}, & \text { if } L(d)_{i, j} \text { is unavailable } \\ 1-\left(1-\frac{\left(T_{M}^{\mathrm{ini}}-T_{M}^{\mathrm{ela}}\right)-V_{M}^{\mathrm{to}}}{\left(T_{M}^{\mathrm{in}}-T_{M}^{\mathrm{ela}}\right)}\right)^{C_{M}}, & \text { else if }\left(D_{j, d} \geq R_{d}\right) \\ 1-\left(1-\frac{\left(T_{M}^{\mathrm{ini}}-T_{M}^{\mathrm{ela}}\right)-V_{M}^{\mathrm{in}}}{\left(T_{M}^{\mathrm{ini}}-T_{M}^{\text {ela }}\right)}\right)^{C_{M}}, & \text { else if }\left(D_{j, d}<R_{d}\right) .\end{cases}
$$

2) Message Transmission: It is observed that the message with the largest value of $1-\left(1-\left(\left(T_{M}^{\mathrm{ini}}-T_{M}^{\mathrm{ela}}\right)-V_{M}^{\mathrm{in}}\right) /\left(T_{M}^{\mathrm{ini}}-\right.\right.$ $\left.\left.T_{M}^{\text {ela }}\right)\right)^{C_{M}}$ is considered with the highest priority for transmission. However, if we consider the case to transmit the message with the negative value of $1-\left(1-\left(\left(T_{M}^{\text {ini }}-T_{M}^{\text {ela }}\right)-V_{M}^{\text {in }}\right) /\right.$ $\left.\left(T_{M}^{\mathrm{ini}}-T_{M}^{\text {ela }}\right)\right)^{C_{M}}$, prior to those with the positive value of $1-\left(1-\left(\left(T_{M}^{\mathrm{ini}}-T_{M}^{\mathrm{ela}}\right)-V_{M}^{\mathrm{to}}\right) /\left(T_{M}^{\mathrm{ini}}-T_{M}^{\mathrm{ela}}\right)\right)^{C_{M}}$, the former message may get expired before being delivered to its destination, although it is within the movement range estimated for the destination.

Considering those sprayed without knowledge of $N_{d}$, the messages replicated in relation to the movement range estimated for $N_{d}$ are considered to be more important. This is because it is desirable to transmit the messages with a higher priority knowing where their destinations are. As an example, it is shown in Fig. 5 that $N_{i}$ and $N_{j}$ are unaware of destination $N_{d 3}$ for $M 3$ and $N_{d 4}$ for $M 4$. Assuming that $P_{M 1}=1-$ $\left(1-\left(\left(T_{M 1}^{\mathrm{ini}}-T_{M 1}^{\mathrm{ela}}\right)-V_{M 1}^{\mathrm{to}}\right) /\left(T_{M 1}^{\mathrm{ini}}-T_{M 1}^{\mathrm{ela}}\right)\right)^{C_{M 1}}=3, P_{M 2}=$ $1-\left(1-\left(\left(T_{M 2}^{\mathrm{ini}}-T_{M 2}^{\mathrm{ela}}\right)-V_{M 2}^{\mathrm{in}}\right) /\left(T_{M 2}^{\mathrm{ini}}-T_{M 2}^{\mathrm{ela}}\right)\right)^{C_{M 2}}=1, P_{M 3}=$ $1-\left(1-\left(T_{M 3}^{\text {ini }}-T_{M 3}^{\text {ela }}\right) / T_{M 3}^{\text {ini }}\right)^{C_{M 3}}=4$, and $P_{M 4}=1-(1-$ $\left.\left(T_{M 4}^{\mathrm{ini}}-T_{M 4}^{\mathrm{ela}}\right) / T_{M 4}^{\mathrm{ini}}\right)^{C_{M 4}}=2$, respectively, then $M 1$ is

\footnotetext{
${ }^{7}$ For example, $V_{M}^{\text {to }}$ and $V_{M}^{\text {in }}$ for some copies of a message may not be updated due to rare encounter opportunity. Therefore, the $P_{M}$ of those message copies may not be the most recent value.
} 


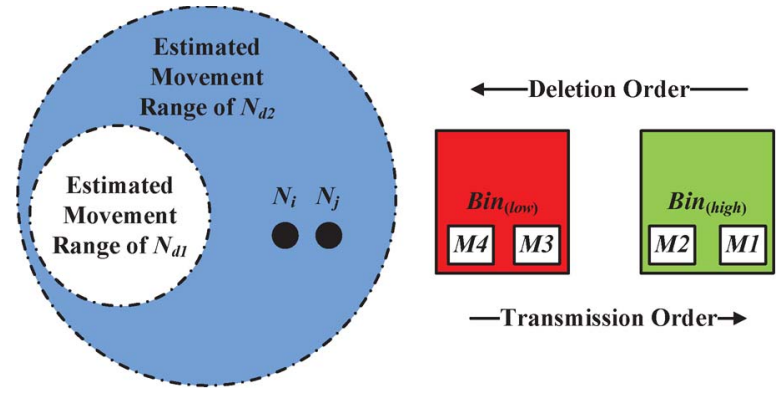

Fig. 5. Example of message management.

considered with the highest priority for transmission, although $N_{j}$ is out of the movement range estimated for $N_{d 1}$.

\section{Algorithm 4 Message Management}

1: for each encounter between $N_{i}$ and $N_{j}$ do

2: both of them update the ID of the delivered messages

3: both of them delete the copies of the delivered messages from their buffer space

4: for each message $M$ replicated to $N_{i}$ do

5: $\quad$ if both $N_{i}$ and $N_{j}$ have knowledge about $N_{d}$ then

6: $\quad P_{M}$ is calculated based on the cases, where $\left(D_{j, d} \geq\right.$ $\left.R_{d}\right)$ or $\left(D_{j, d}<R_{d}\right)$

7: $\quad$ transmit $M$ according to the descending order of $P_{M}$

8: $\quad$ else if neither $N_{i}$ nor $N_{j}$ has encountered $N_{d}$ in the past then

9: $\quad P_{M}$ is calculated based on the case where $L(d)_{i, j}$ is unavailable

10: $\quad$ transmit $M$ according to the descending order of $P_{M}$

11: $\quad$ end if

12: end for

13: if $N_{j}$ does not have sufficient buffer space to receive $M$ then

14: $\quad$ if neither $N_{i}$ nor $N_{j}$ has knowledge about $N_{d}$ then

15: $\quad N_{j}$ removes its carried messages with the lowest value of $P_{M}$ from $B i n_{\text {(low) }}$

16: else

17: $\quad N_{j}$ removes its carried messages with the lowest

18: $\quad$ end if value of $P_{M}$ from $\operatorname{Bin}_{(\text {high })}$

19: end if

20: end for

3) Buffer Management: Because of the intermittent connections between nodes in DTNs, each node uses a buffer to store the messages needed to transmit. Here, messages are classified into two bins $\operatorname{Bin}_{(\text {high })}$ and $\operatorname{Bin}_{(\text {low })}$, respectively, considering the awareness of the destination based on the view of its current carrier. For example, if neither $N_{i}$ nor $N_{j}$ did not meet $N_{d}$ in the past, then the $M$ destined to $N_{d}$ is put in $\operatorname{Bin}_{(\text {low })}$.

Since each node may not have sufficient space to store all the received messages, messages classified into $\operatorname{Bin}_{(\text {low })}$ are deleted prior to those classified into $\operatorname{Bin}_{(\mathrm{high})}$, following the same rule discussed for message transmission. Referring to Fig. 5, an example for message deletion is also shown. This is implemented to keep the message where its destination is known, as compared with the case when keeping the message without knowledge about its destination. Regarding the messages classified into the same bin, they are also prioritized according to the definition of $P_{M}$. For example, the message with a negative value of $1-\left(1-\left(\left(T_{M}^{\text {ini }}-T_{M}^{\text {ela }}\right)-\right.\right.$ $\left.\left.V_{M}^{\text {in }}\right) /\left(T_{M}^{\text {ini }}-T_{M}^{\text {ela }}\right)\right)^{C_{M}}$ is deleted prior to those with a positive value of $1-\left(1-\left(\left(T_{M}^{\mathrm{ini}}-T_{M}^{\mathrm{ela}}\right)-V_{M}^{\mathrm{to}}\right) /\left(T_{M}^{\mathrm{ini}}-T_{M}^{\mathrm{ela}}\right)\right)^{C_{M}}$, although the former has already been within the movement range estimated for the destination.

If a message copy is successfully delivered, it is essential to delete other copies of this message in the network, to free the buffer space for other undelivered messages. In this case, each node maintains a list to record the IDs of delivered messages in the network, then exchanges and updates the information in this list. Note that a node carrying the copy of the delivered message may not receive this knowledge in time, but the node will finally receive it with high probability because of the flooding nature of the acknowledgement information. In the worst case that a node without this knowledge will constantly carry the delivered message copy until the destination node is in proximity, the destination will delete the copy since it has been already received.

\section{F. Discussion on Storage for Information Update}

First, the threshold values $V_{M}^{\text {to }}, V_{M}^{\text {aw }}$, and $V_{M}^{\text {in }}$ are the flags recorded in each data message. The size of each flag is very small compared with the size of data itself. Second, we do envision infinite buffer space for main evaluation, considering the nature of DTNs that nodes always store messages until a new encounter is available for message relay, known as SCF. Therefore, the size of the routing table can be ignored compared with data messages stored in each node. Concerning the storage overhead of maintaining the updated routing information, it is $O\left(K^{2}\right)$, where the number of nodes in the network is denoted as $K$, similar to other works using history information for making a routing decision. For real implementation, the structure of the routing table should be a "Map 〈Key, Value $\rangle$ " structure, where "Key" is the nodal ID, and "Value" is a tuple containing historical based location, average moving speed, and encounter time.

\section{Performance Evaluation}

The performance is evaluated using the Opportunistic Network Environment (ONE) [28] version 1.4.1, which is a wellknown Java-based simulator that particularly contributes to the research on DTN routing. The main evaluation is based on the Helsinki city scenario shown in Fig. 6(a).

Considering that the map route has an effect on the performance of geographic routing assuming the continuous moving direction for prediction, we select other two city scenarios from real world shown in Fig. 6(b) and (c), respectively, via OpenStreetMap, and convert them into the WKT format interpreted by ONE. The mobile node chooses the shortest 


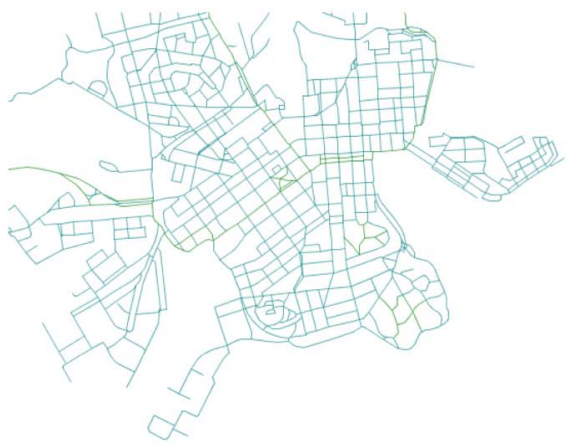

(a)

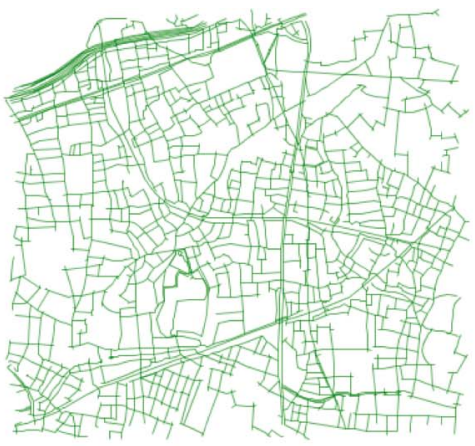

(b)

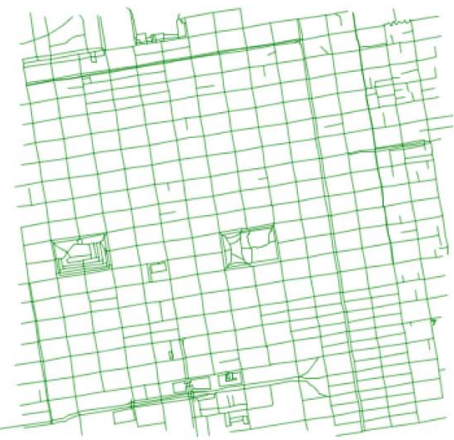

(c)

Fig. 6. Snapshots of the Helsinki City, Tokyo City, and San Francisco City scenarios. (a) Helsinki City; $4500 \mathrm{~m} \times 3400 \mathrm{~m}$. (b) Tokyo City; $2500 \mathrm{~m} \times 2500 \mathrm{~m}$. (c) San Francisco City; $2500 \mathrm{~m} \times 2500 \mathrm{~m}$.

path to a randomly selected place via the Dijkstra shortest path scheme, based on their current location and moving speed. Under city scenarios, 100 mobile nodes are configured with the uniformly varied $1-10-\mathrm{m} / \mathrm{s}$ moving speed along a generated path. We aim to examine how the variation of the nodal moving speed affects the performance of the routing scheme. In other words, the nodal moving speed is different, depending on the path that a node is moving in.

For the purpose of generalization that GSaR ideally assumes a constant moving speed without movement restriction, we also select a $1000 \times 1000 \mathrm{~m}^{2}$ random waypoint (RWP) scenario, where 100 mobile nodes with $5-5-\mathrm{m} / \mathrm{s}$ constant moving speed are configured. Note that mobile nodes move randomly and freely without restrictions in RWP, as compared with the city scenario where the nodal movement is restricted by the map route. In RWP, each node moves along a zigzag line from one waypoint to the next, where the waypoints are uniformly distributed over the given area. The initial distribution of mobile nodes under all scenarios varies based on the corresponding simulation speed.

The communication technique ${ }^{8}$ is configured as $2-\mathrm{Mb} / \mathrm{s}$ bandwidth and 10-m transmission range, which is referred to in [7] and [8] considering the communication between those shortrange devices, e.g., people with mobile phones in vehicles. Note that the network is sparse and highly dynamic, since the number of connectivities is small as compared with network area, considering the given speed configuration and transmission range. Messages are propagated via opportunistic behavior, as bridged by the lightweight mobile nodes via Bluetooth. In our simulations, the message size is set to a large value, envisioned for large file transmission in DTNs. Messages are set with 90-min lifetime, 30-s generation interval, and 500-KB size, generated before $27000 \mathrm{~s}$ with additional $90 \times 60=5400 \mathrm{~s}$ to consume the unexpired messages.

Given the above default configurations, GSaR is compared with AaR [7], CaD [8], SaF [12], LSF [13], and PROPHET [17], where the time window $W$ in GSaR is set with $5 \mathrm{~s}$. The

\footnotetext{
${ }^{8}$ In DTNs, knowledge of nodes in proximity is essential before initiating any kind of data transfer. For realistic application, a short-range WiFi is an alternative to Bluetooth within a large area, compared with the size of scenarios we use in simulation. The interval and duration of discovery can be adjusted based on the current node's location and the location of nodes it has encountered.
}

default value $L$ for all the spray-based schemes is configured as 10 , referring to [12] that choosing $L$ is equal to about $10 \%$ of the total number of nodes in the network. The performance is evaluated in terms of varied message lifetime, generation interval, varied maximum moving speed, and value of $L$, given infinite buffer space. The effect of limited buffer space is also shown for further comparison. To provide average results, this $32400 \mathrm{~s}=9 \mathrm{~h}$ simulation is independently run with different speeds and shown with $95 \%$ confidence interval.

We further import an energy model for all the evaluated routing schemes to show their energy consumption. Here, the initial energy of the battery for mobile nodes is fixed as 3600000 units, and energy consumed for transmission is fixed as 100 units per $0.1 \mathrm{~s}$. Note that the energy might be wasted due to the unsuccessful transmission, where only a proportion of message size has been transmitted if the connectivity is disrupted. Then, the product of the time duration for transmitting this proportion and the energy consumed is also taken into account. The main purpose of evaluating the energy consumption is to show the fairness of routing schemes. Note that we do not account for the energy consumption for nodal discovery and receiving, as their values are the same for all evaluated routing schemes. Here, only the energy consumption in relation to the number of times a node relays messages is shown as result. A good fairness means the resource, such as energy used by each node to relay messages, is equal.

The evaluation metrics are explained as follows.

- Delivery Ratio: This is the ratio between the number of messages delivered and the total number of messages generated.

- Average Delivery Latency: This is the average delay for the messages to be delivered from the source node to the destination.

- Overhead Ratio: This is the ratio between the number of relayed messages (excluding the delivered messages) and the number of delivered messages.

\section{A. Influence of Message Lifetime}

We observe that GSaR outperforms other schemes in Fig. 7(a) particularly given short message lifetime. Here, GSaR overcomes the local maximum problem that happens in each case, which further increases the possibility that messages can 


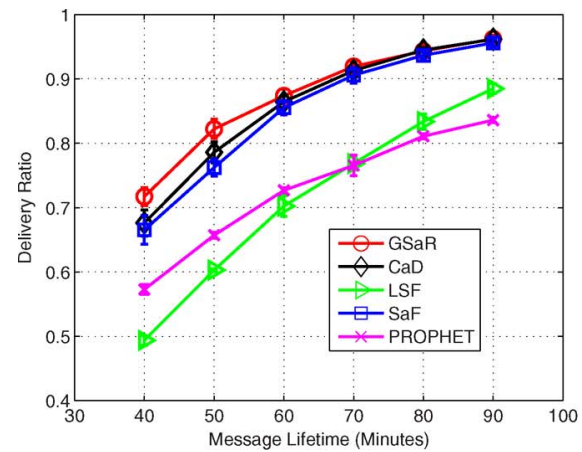

(a)

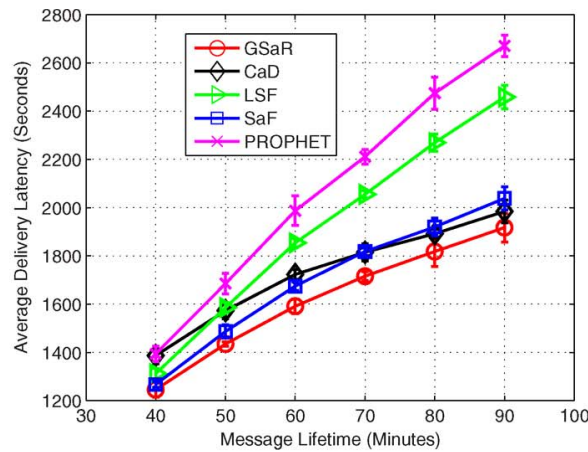

(b)

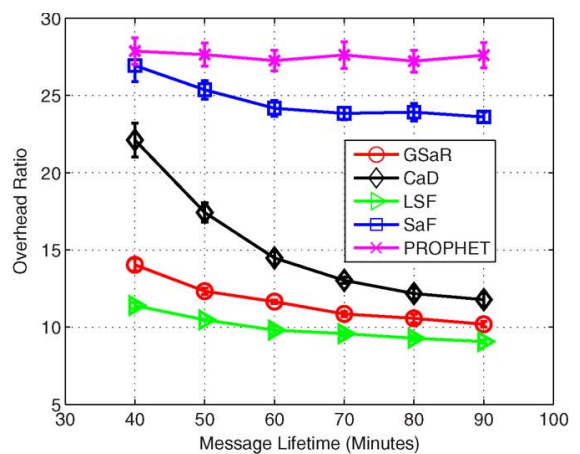

(c)

Fig. 7. Influence of message lifetime. (a) Delivery ratio. (b) Average delivery latency. (c) Overhead ratio.

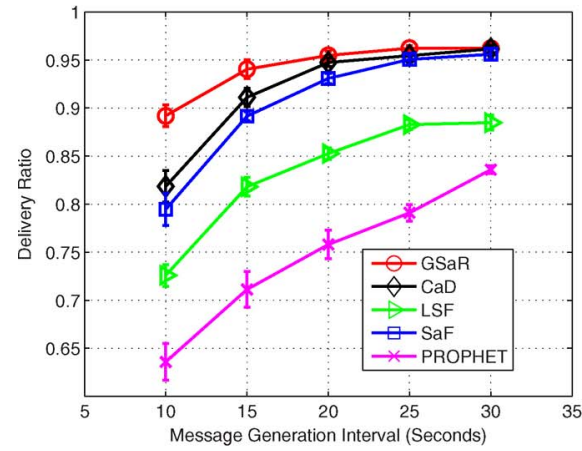

(a)

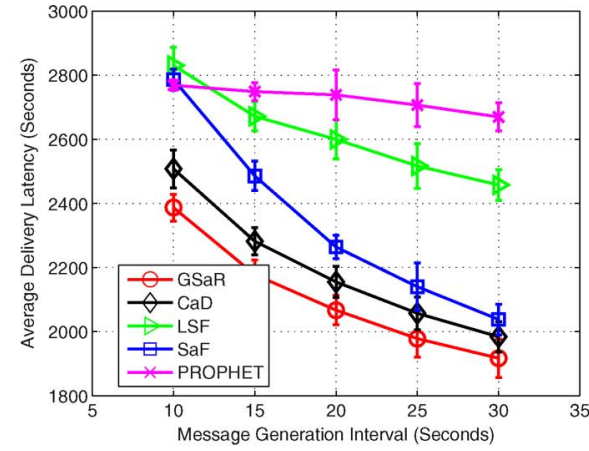

(b)

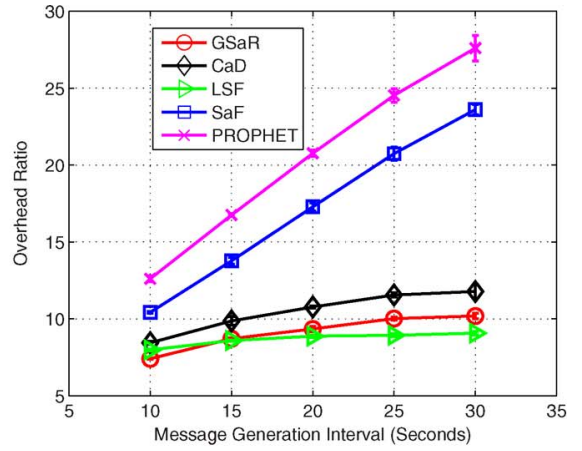

(c)

Fig. 8. Influence of message generation interval. (a) Delivery ratio. (b) Average delivery latency. (c) Overhead ratio.

be delivered within the expiration deadline. In particular, $\mathrm{CaD}$ begins to outperform $\mathrm{SaF}$ given the increased message lifetime. This is because although the redundant message replications in $\mathrm{CaD}$ are reduced by using $\mathrm{DF}$, messages with short lifetimes might not be delivered if using lesser number of message copies. Since mobile nodes are with high speed, SaF also performs well in this case given the nature of the spray-based routing scheme, which is relying on the highly mobile node to deliver messages only via the limited number of copies. Here, the reason LSF performs worst given the short message lifetime is that replicating only a limited number of message copies is insufficient for delivery.

Meanwhile, GSaR keeps the lowest average delivery latency in Fig. 7(b). Here, the slope of this performance implies the capability of a routing decision, where PROPHET estimating the encounter probability based on the frequency, performs worst due to not considering the recent encounter time as adopted by LSF. The contribution from handling the local maximum problem also reduces the delivery latency by promoting message spraying, particularly if the message is close to its expiration deadline.

In Fig. 7(c), GSaR and $\mathrm{CaD}$ benefit from a significantly decreased overhead ratio given the increased message lifetime, as compared with $\mathrm{SaF}$ and PROPHET. Since the number of message replications is unlimited in $\mathrm{CaD}$, more message copies are replicated even if they may expire soon. In light of this, $\mathrm{CaD}$ is with the dramatically decreased overhead ratio following an increased message lifetime. Although $\mathrm{SaF}$ achieves a close delivery ratio compared with $\mathrm{GSaR}$ and $\mathrm{CaD}$, its overhead ratio is higher than these two geographic routing schemes, due to forwarding messages based on the unstable topology-based encounter history.

\section{B. Influence of Message Generation Interval}

In Fig. 8(a), all the schemes benefit from the alleviated bandwidth contention by achieving the increased delivery ratio. This is different from $\mathrm{CaD}$ without limiting the number of message copies, where the bandwidth contention becomes dramatically in case of a 10-s generation interval.

GSaR also maintains the lowest average delivery latency in Fig. 8(b). Compared with geographic routing scheme CaD, PROPHET does not achieve a significantly decreased average delivery latency, due to using the utility metric in relation to encounter frequency to make a routing decision and message transmission. It is highlighted that the spray-based routing schemes, such as GSaR, SaF, and LSF, obtain a dramatically decreased average delivery latency. This is because replicating a small number of message copies does not result in too much bandwidth contention. The observation in Fig. 8(b) implies that it is more effective to consider the nodal mobility and message lifetime for making a routing decision and transmission, in addition to limiting the number of message replications for efficient delivery.

Although both GSaR and $\mathrm{CaD}$ handle the local maximum problem, they achieve a smoother slope regarding overhead ratio in Fig. 8(c), due to the nature of DF to reduce the replication redundancy. Meanwhile, since LSF limits the number 


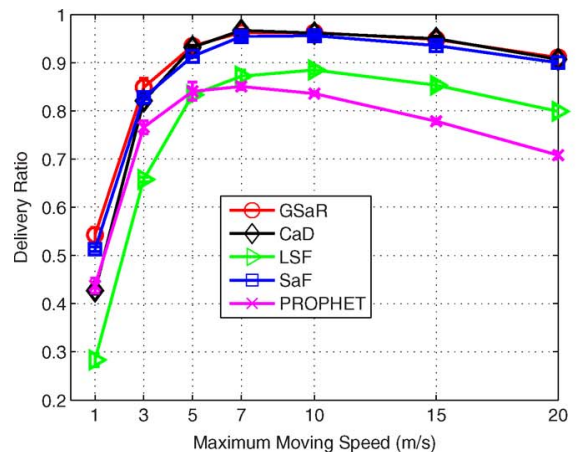

(a)

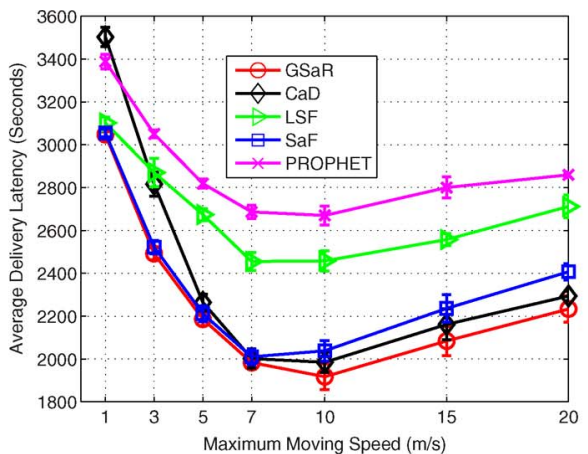

(b)

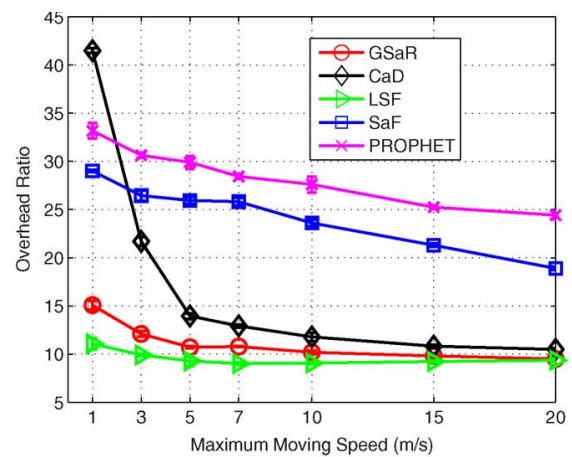

(c)

Fig. 9. Influence of maximum moving speed. (a) Delivery ratio. (b) Average delivery latency. (c) Overhead ratio.

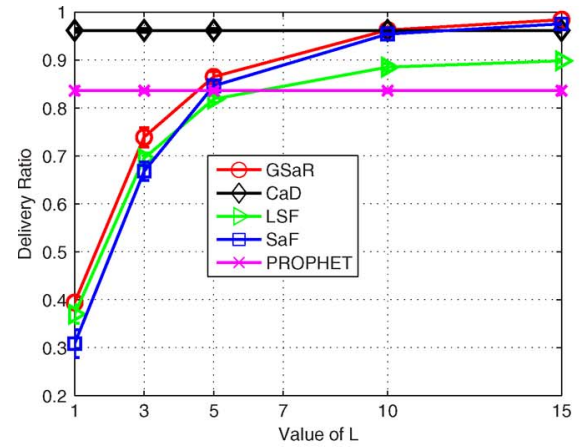

(a)

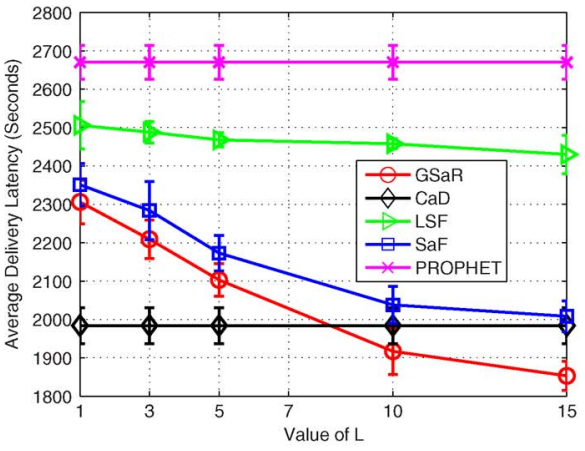

(b)

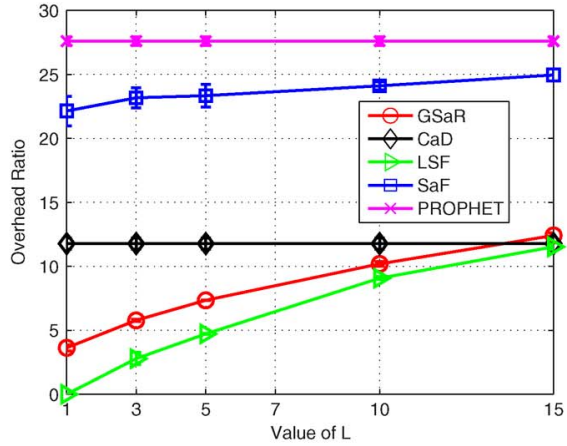

(c)

Fig. 10. Influence of initial copy ticket value. (a) Delivery ratio. (b) Average delivery latency. (c) Overhead ratio.

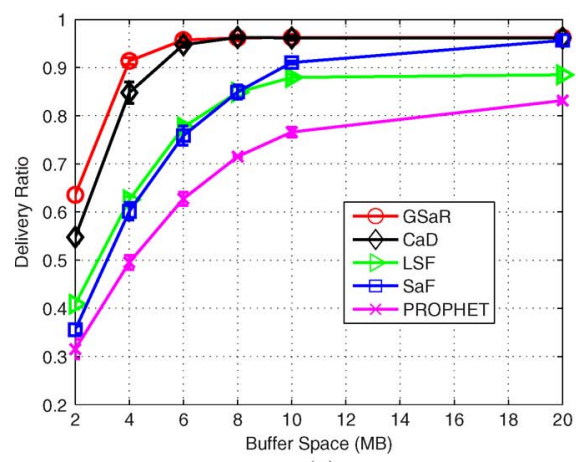

(a)

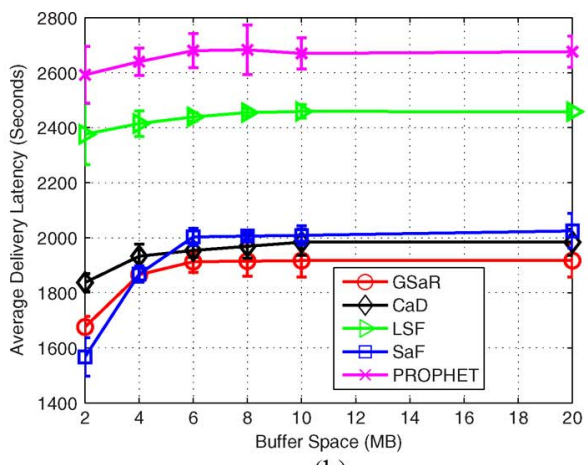

(b)

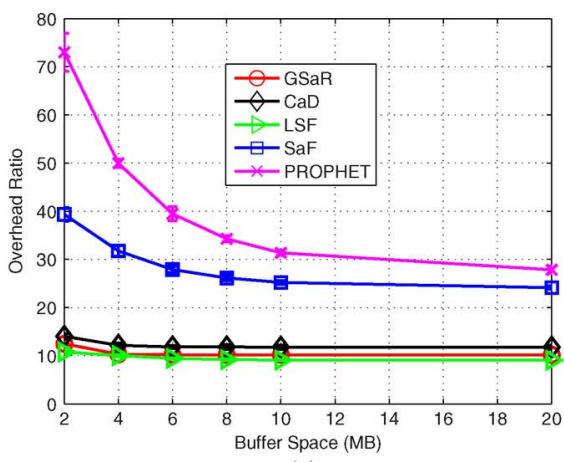

(c)

Fig. 11. Influence of buffer space. (a) Delivery ratio. (b) Average delivery latency. (c) Overhead ratio.

of message replications to be $(L-1)$ only, it is with the lowest overhead ratio.

\section{Influence of Maximum Moving Speed}

In one case, if increasing the maximum moving speed with a moderate level, the message delivery ratio is accordingly increased because the high nodal mobility yields more encounter opportunities. In another case, a further aggressively high moving speed inevitably reduces the encounter duration, as such all the schemes suffer from a decreased delivery ratio. With this in mind, in Fig. 9(a), all the schemes first obtain the increased delivery ratio if the maximum moving speed is varied up to $7 \mathrm{~m} / \mathrm{s}$, whereas their performance is degraded if the maximum moving speed is faster. Without forwarding message with $\left(C_{M}=1\right)$ copy ticket, LSF performs worst if mobile nodes are not highly mobile, as indicated when the maximum moving speed is slower than $5 \mathrm{~m} / \mathrm{s}$.

Due to a moderately high mobility that helps diffuse message copies, the average delivery latency is decreased for all the routing schemes. However, a further increased average delivery latency happens herein, because the encounter duration between pairwise nodes is insufficient to successfully transmit all the replicated messages. In light of this, all the schemes in Fig. 9(b) suffer from a fluctuation regarding this performance metric, where GSaR keeps the lowest level.

GSaR also achieves the lowest overhead ratio in Fig. 9(c), where the local maximum problem in the spray phase is prevented, if mobile nodes are fast enough. This is because by using a smaller value of $V_{M}^{\text {to }}$ and $V_{M}^{\text {in }}$ to compare with 
TABLE III

COMPARISON BETWEEN GSaR AND AaR

\begin{tabular}{|l|l|l|l|l|l|l|}
\hline \multirow{2}{*}{} & \multicolumn{2}{|l|}{ Delivery Ratio } & \multicolumn{2}{l|}{$\begin{array}{l}\text { Average } \\
\text { Delivery } \\
\text { Latency }\end{array}$} & \multicolumn{2}{l|}{ Overhead Ratio } \\
\cline { 2 - 7 } & GSaR & AaR & GSaR & AaR & GSaR & AaR \\
\hline \hline Default & 0.962 & 0.990 & $1917 \mathrm{~s}$ & $1902 \mathrm{~s}$ & 10.19 & 20.48 \\
Configuration & $( \pm 0.006)$ & $( \pm 0.001)$ & $( \pm 60)$ & $( \pm 17)$ & $( \pm 0.19)$ & $( \pm 0.36)$ \\
\hline 10s Generation & 0.892 & 0.865 & $2387 \mathrm{~s}$ & $2426 \mathrm{~s}$ & 7.40 & 8.81 \\
Interval & $( \pm 0.011)$ & $( \pm 0.011)$ & $( \pm 42)$ & $( \pm 73)$ & $( \pm 0.02)$ & $( \pm 0.05)$ \\
\hline 2MB Buffer & 0.636 & 0.634 & $1676 \mathrm{~s}$ & $1588 \mathrm{~s}$ & 12.47 & 31.95 \\
Space & $( \pm 0.014)$ & $( \pm 0.006)$ & $( \pm 39)$ & $( \pm 30)$ & $( \pm 0.24)$ & $( \pm 0.27)$ \\
\hline 1m/s & 0.543 & 0.515 & $3048 \mathrm{~s}$ & $3321 \mathrm{~s}$ & 15.08 & 36.02 \\
Maximum & $( \pm 0.014)$ & $( \pm 0.015)$ & $( \pm 14)$ & $( \pm 42)$ & $( \pm 0.44)$ & $( \pm 0.25)$ \\
Moving Speed & & & & & & \\
\hline 20m/s & 0.911 & 0.953 & $2233 \mathrm{~s}$ & $2370 \mathrm{~s}$ & 9.50 & 15.83 \\
Maximum & $( \pm 0.008)$ & $( \pm 0.002)$ & $( \pm 62)$ & $( \pm 23)$ & $( \pm 0.13)$ & $( \pm 0.06)$ \\
Moving Speed & & & & & & \\
\hline
\end{tabular}

$\left(T_{M}^{\mathrm{ini}}-T_{M}^{\mathrm{ela}}\right)$, a lesser number of message copies are sprayed. In contrast, considering that $\mathrm{CaD}$ is without limiting the number of message replications, a slow moving speed yields more message copies to be replicated given a local maximum problem. Although $\mathrm{CaD}$ also reduces the message replication to the candidate node via DF, the slow nodal movement is unhelpful for message delivery. Therefore, $\mathrm{CaD}$ suffers from the highest overhead ratio given $1-\mathrm{m} / \mathrm{s}$ maximum moving speed, because it makes more message replications, but most of them are not delivered.

\section{Influence of Initial Copy Ticket Value}

We observe that LSF outperforms $\mathrm{SaF}$ at the initial stage in Fig. 10(a). This is because when the number of message copies is very small, selecting the candidate node that has met the destination more recently, as performed by LSF, increases the message delivery potential. SaF performs worse than LSF given a small $L$, because the former sprays message without selecting the candidate node. Here, GSaR outperforms LSF and SaF, by using historical geographic information to estimate the delivery potential of the mobile node, rather than the historical topologybased encounter information under high dynamic scenario. If continually increasing the value of $L, \mathrm{SaF}$ begins to outperform LSF. This is because a larger $L$ contributes to a higher possibility that one of the sprayed $(L-1)$ message copies can be delivered in a timely way, particularly if with it is the assistance of a forwarding scheme adopted by SaF.

In Fig. 10(b), the average delivery latency is reduced by spraying more message copies. It is observed that GSaR and $\mathrm{SaF}$ benefit more from the increased $L$, due to further forwarding message with $\left(C_{M}=1\right)$ copy ticket, as compared with LSF, which only relies on the direct encounter between the destination to deliver this given message. The advantage of using a geographic routing scheme is also indicated herein, as GSaR can achieve the lowest average delivery latency given the small $L$. Furthermore, LSF suffers from a smooth decreased average delivery latency, due to not using the nodal mobility to relay the message with the $\left(C_{M}=1\right)$ copy ticket.

In Fig. 10(c), all the spray-based schemes suffer from the increased overhead ratio for delivering more messages. In GSaR, as the number of message being relayed is higher than $(L-1)$, the overhead ratio of GSaR is slightly higher than LSF. Note that since $\mathrm{SaF}$ does not consider the stable convergence under
TABLE IV

INFLUENCE OF HANDLING THE LOCAL MAXIMUM PROBLEM

\begin{tabular}{|c|c|c|c|c|c|c|}
\hline \multirow[t]{2}{*}{$\begin{array}{l}\text { Message } \\
\text { Lifetime }\end{array}$} & \multicolumn{2}{|c|}{ Delivery Ratio } & \multicolumn{2}{|c|}{$\begin{array}{l}\text { Average } \\
\text { Delivery } \\
\text { Latency }\end{array}$} & \multicolumn{2}{|c|}{ Overhead Ratio } \\
\hline & GSaR & $\begin{array}{l}\text { GSaR } \\
\text { (WH) }\end{array}$ & GSaR & $\begin{array}{l}\text { GSaR } \\
(\mathrm{WH})\end{array}$ & GSaR & $\begin{array}{l}\text { GSaR } \\
(\mathrm{WH})\end{array}$ \\
\hline $\begin{array}{l}40 \\
\text { Minutes }\end{array}$ & $\begin{array}{l}0.717 \\
( \pm 0.014)\end{array}$ & $\begin{array}{l}0.651 \\
( \pm 0.002)\end{array}$ & $\begin{array}{l}1246 \mathrm{~s} \\
( \pm 31)\end{array}$ & $\begin{array}{l}1224 \mathrm{~s} \\
( \pm 35)\end{array}$ & $\begin{array}{l}14.04 \\
( \pm 0.40)\end{array}$ & $\begin{array}{l}12.60 \\
( \pm 0.17)\end{array}$ \\
\hline $\begin{array}{l}90 \\
\text { Minutes }\end{array}$ & $\begin{array}{l}0.962 \\
( \pm 0.006)\end{array}$ & $\begin{array}{l}0.945 \\
( \pm 0.010)\end{array}$ & $\begin{array}{l}1917 \mathrm{~s} \\
( \pm 60)\end{array}$ & $\begin{array}{l}1929 \mathrm{~s} \\
( \pm 57)\end{array}$ & $\begin{array}{l}10.19 \\
( \pm 0.19)\end{array}$ & $\begin{array}{l}9.67 \\
( \pm 0.11)\end{array}$ \\
\hline
\end{tabular}

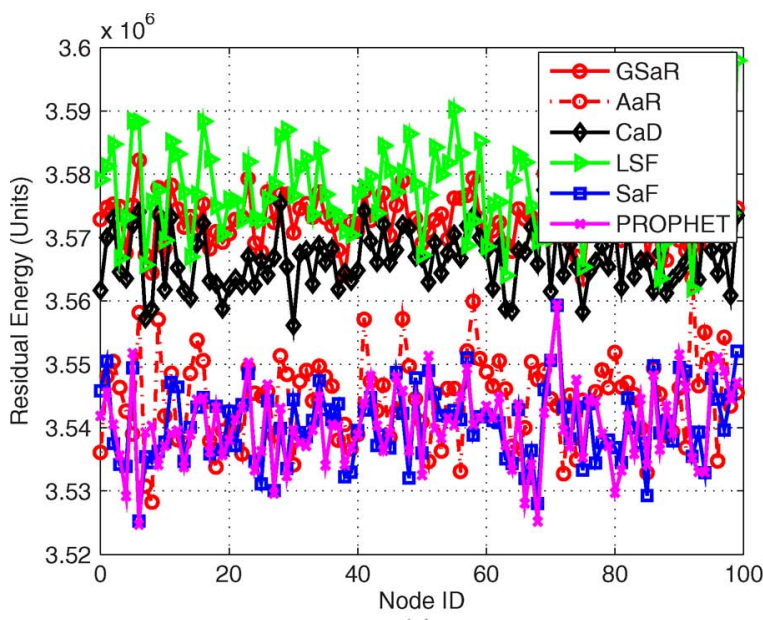

(a)

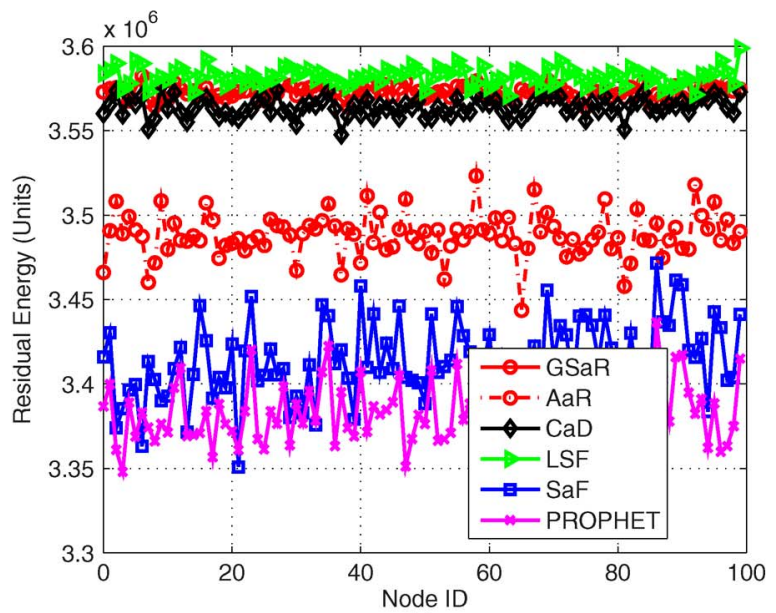

(b)

Fig. 12. Energy distribution. (a) 10-m transmission range. (b) 50-m transmission range.

high dynamic scenario, the message with $\left(C_{M}=1\right)$ copy ticket is forwarded with redundancy. As such, $\mathrm{SaF}$ suffers from a higher overhead ratio than LSF and GSaR.

\section{E. Influence of Buffer Space}

In Fig. 11(a), we observe that GSaR achieves the highest delivery ratio, particularly given a small buffer space. One reason is that GSaR only sprays a limited number of message copies into the network; thus, the buffer occupation for message copies is less than that consumed by $\mathrm{CaD}$ and PROPHET. By comparing with $\mathrm{SaF}$ and $\mathrm{LSF}$, another reason is that GSaR deletes the message with the least delivery potential if buffer 


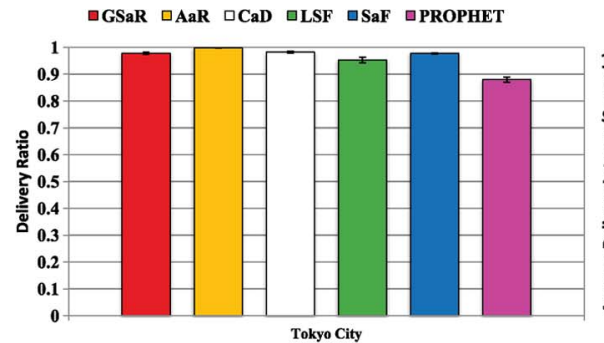

(a)

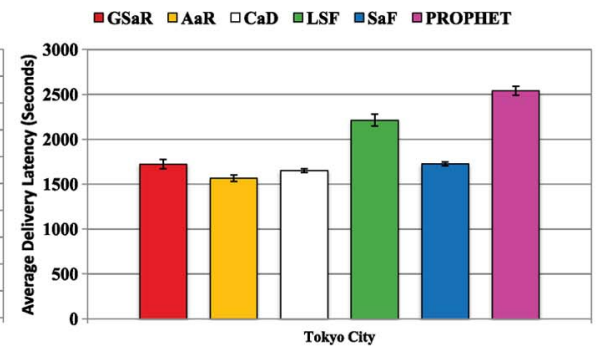

(b)

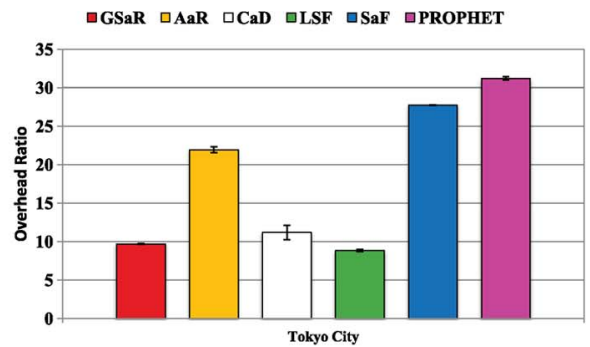

(c)

Fig. 13. Performance under the Tokyo City scenario with Default configuration. (a) Delivery ratio. (b) Average delivery latency. (c) Overhead ratio.

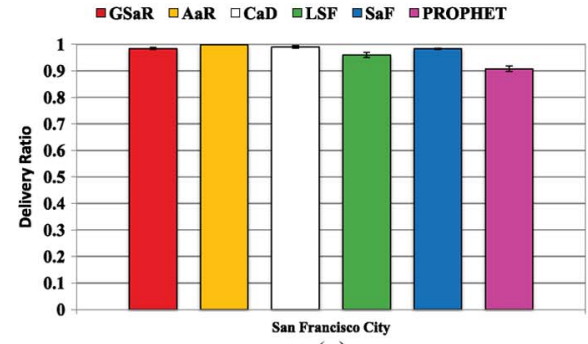

(a)

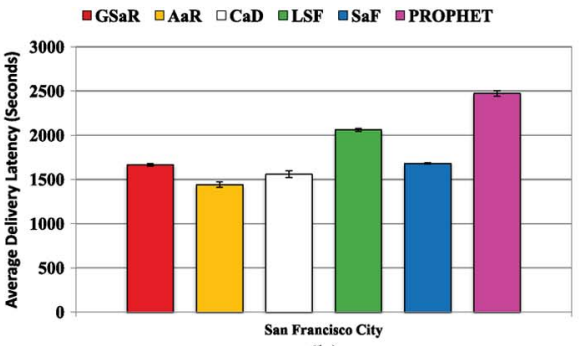

(b)

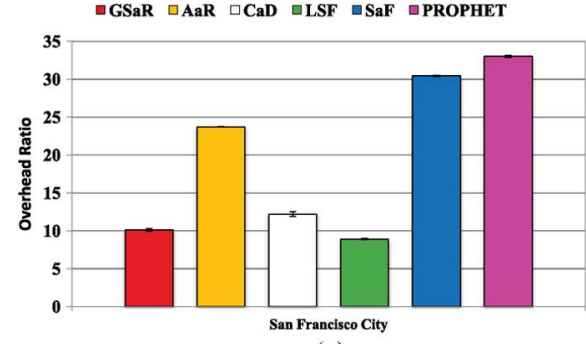

(c)

Fig. 14. Performance under the San Francisco City scenario with default configuration. (a) Delivery ratio. (b) Average delivery latency. (c) Overhead ratio.

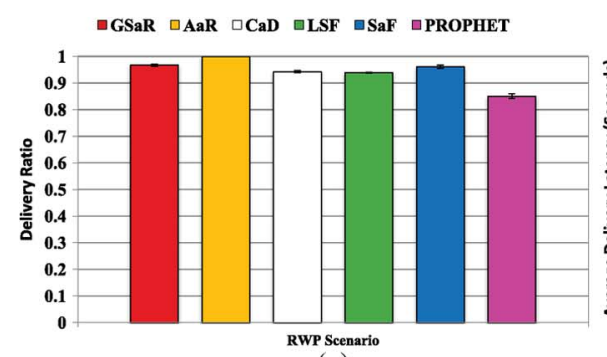

(a)

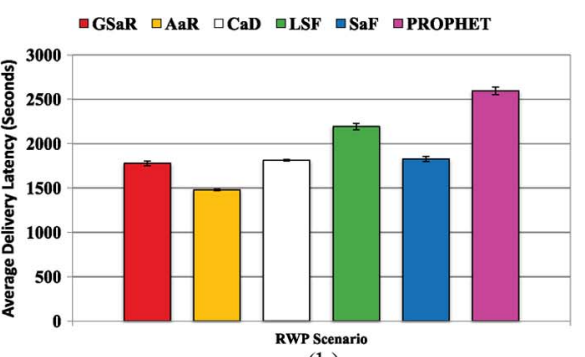

(b)

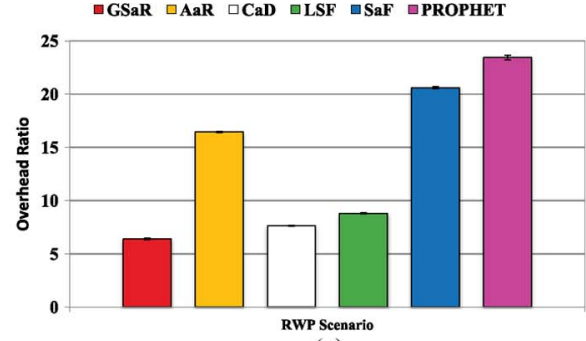

(c)

Fig. 15. Performance under the RWP scenario with default configuration. (a) Delivery ratio. (b) Average delivery latency. (c) Overhead ratio.

overflows. Note that in GSaR, the delivery potential is estimated considering the number of copies, historical nodal movement, and message lifetime. Different from the observation given infinite buffer space in Fig. 7(a), SaF performs worse than LSF given the small buffer space herein. As previously indicated, the buffer space of some mobile nodes might be exhausted in $\mathrm{SaF}$, due to always forwarding the message with the $\left(C_{M}=1\right)$ copy ticket to the nodes with an unstable utility metric. This short-term behavior results in aggressive message deletion.

In Fig. 11(b), since a larger buffer space increases the possibility that messages would survive in the network, all the schemes are with an increased average delivery latency. Although $\mathrm{SaF}$ achieves a lightly lower average delivery latency than GSaR when the buffer space is smaller than $6 \mathrm{MB}$, the former is with a lower delivery ratio [see Fig. 11(a)].

By using DF, GSaR and CaD benefit from a lower overhead ratio in Fig. 11(c), with a smooth decreased slope. This is because a large number of message copies results in aggressive contention of buffer space, whereas the deleted message copies still require future replication and transmission. In light of this, PROPHET and SaF perform worse than other schemes, due to either without limiting the number of message copies or without using DF to control redundancy. Since the number of message replications is limited up to $(L-1)$ in LSF, it is with the lowest overhead ratio.

\section{F. Comparison Between GSaR and AaR}

From the results in Table III, we observe that GSaR as a spray-based routing scheme achieves a close delivery ratio and average delivery latency as the utility-replication-based scheme AaR. This is because GSaR replicates each message to a small number of candidate nodes, as compared with AaR that makes message replication to each better candidate node greedily selected. Therefore, the latter yields more message copies, which degrades the performance given limited bandwidth and buffer space. Similar to $\mathrm{CaD}, \mathrm{AaR}$ is also without the design when the historical geographic information is unavailable; as such, GSaR outperforms AaR given slow moving speed.

\section{G. Influence of Handling the Local Maximum Problem}

In Table IV, the comparison between GSaR and GSaR (WH) is provided. In GSaR, messages are delivered by using more 
independent paths, due to handling the local maximum problem. Thus, it outperforms GSaR (WH) by delivering messages faster before the expiration deadline, based on the discussed property of the spray phase.

\section{H. Discussion Regarding Fairness and Energy Consumption}

Fairness is a measurement of the energy distribution over the different mobile nodes. In case of the 10-m transmission range, as shown in Fig. 12(a), AaR consumes much energy than GSaR and $\mathrm{CaD}$, due to the local greedy nature in selecting any candidate node with a better delivery potential than current message carrier. Furthermore, GSaR guarantees the lowest overall energy consumption and a relatively fair distribution over mobile nodes, particularly comparing with AaR. In Fig. 12(b), when increasing the transmission range to $50 \mathrm{~m}$, AaR suffers from a large variation regarding energy distribution. In both cases, LSF maintains the highest residual energy due to replicating a message up to $L$ times only, in spite of a lower delivery ratio, particularly given a $10-\mathrm{m}$ transmission range.

\section{Performance Under Other Scenarios}

Given the results under other scenarios, GSaR achieves a high delivery ratio as close to that achieved by $\mathrm{AaR}, \mathrm{CaD}$, and $\mathrm{SaF}$ in Figs. 13(a)-15(a), respectively, similar to previous results under the Helsinki city scenario. Note that since these three scenarios are with a smaller area, LSF achieves a higher delivery ratio given that nodal mobility is able to travel the entire area of these scenarios faster. Meanwhile, GSaR achieves the lowest overhead ratio in Figs. 13(c)-15(c), although its average delivery latency is higher than AaR in Figs. 13(b)-15(b). Note that by limiting the number of message replications, GSaR achieves a lower overhead ratio than $\mathrm{CaD}$.

\section{CONCLUSION}

This paper has investigated the research on geographic routing in DTNs. The proposed GSaR is based on the spray-based routing nature, by only creating a limited number of message copies in the network for efficient delivery. Considering that the real-time location of the mobile destination is unavailable due to sparse network density, GSaR estimates a movement range of the destination via its historical location and average moving speed recorded in the past. Mainly, the spray and relay phases are performed to expedite message copies being sprayed toward this range, postpone their being sprayed out of this range, and prevent them from being sprayed away from this range. Furthermore, the combination of them is based on the investigation of DF to overcome the limitation of a routing decision and handling the local maximum problem. GSaR is evaluated with the design of message management to perform given the limited bandwidth and buffer space. Compared with existing routing schemes, one advantage of GSaR is the efficiency in terms of a low overhead ratio given high delivery ratio. Another advantage is a fair distribution of the lowest energy consumption over the mobile nodes in the network.

\section{REFERENCES}

[1] Y. Cao and Z. Sun, "Routing in delay/disruption tolerant networks: A taxonomy, survey and challenges," IEEE Commun. Surveys Tuts., vol. 15, no. 2, pp. 654-677, 2013.

[2] C. Caini, H. Cruickshank, S. Farrell, and M. Marchese, "Delay and Disruption-Tolerant Networking (DTN): An alternative solution for future satellite networking applications," Proc. IEEE, vol. 99, no. 11, pp. 19801997, Nov. 2011.

[3] P. Pereira et al., "From delay-tolerant networks to vehicular delay-tolerant networks," IEEE Commun. Surveys Tuts., vol. 14, no. 4, pp. 1166-1182, 2011.

[4] T. Small and Z. J. Haas, "The shared wireless infostation model: A new ad hoc networking paradigm," in Proc. ACM MobiHoc, Annapolis, MD, USA, Jun. 2003, pp. 233-244.

[5] K. Wei, X. Liang, and K. Xu, "A survey of social-aware routing protocols in delay tolerant networks: Applications, taxonomy and design-related issues," IEEE Commun. Surveys Tuts., vol. 6, no. 1, pp. 556-578, 2014.

[6] B. Karp and H. T. Kung, "GPSR: Greedy perimeter stateless routing for wireless networks," in Proc. ACM MobiCom, Boston, MA, USA, Aug. 2000, pp. 243-254.

[7] Y. Cao, Z. Sun, H. Cruickshank, and F. Yao, "Approach-and-roam: A geographic routing scheme for delay/disruption-tolerant networks," IEEE Trans. Veh. Technol., vol. 63, no. 1, pp. 266-281, Jan. 2014.

[8] Y. Cao, Z. Sun, N. Wang, F. Yao, and H. Cruickshank, "Converge-anddiverge: A geographic routing for delay/disruption-tolerant networks using a delegation replication approach," IEEE Trans. Veh. Technol., vol. 62 , no. 5, pp. 2339-2343, Jun. 2013.

[9] H.-Y. Huang et al., "Performance evaluation of SUVnet with real-time traffic data," IEEE Trans. Veh. Technol., vol. 56, no. 6, pp. 3381-3396, Nov. 2007.

[10] J. Zhu et al., "A mobility prediction-based adaptive data gathering protocol for delay tolerant mobile sensor network," in Proc. IEEE GLOBECOM, New Orleans, LA, USA, Dec. 2008, pp. 1-5.

[11] X. Li, W. Shu, M. Li, H. Huang, and M.-Y. Wu, "DTN routing in vehicular sensor networks," in Proc. IEEE GLOBECOM, New Orleans, LA, USA, Dec. 2008, pp. 1-5.

[12] T. Spyropoulos, K. Psounis, and C. Raghavendra, "Efficient routing in intermittently connected mobile networks: The multiple-copy case," IEEE/ACM Trans. Netw., vol. 16, no. 1, pp. 77-90, Feb. 2008.

[13] T. Spyropoulos, T. Turletti, and K. Obraczka, "Routing in delay-tolerant networks comprising heterogeneous node populations," IEEE Trans. Mobile Comput., vol. 8, no. 8, pp. 1132-1147, Aug. 2009.

[14] V. Soares, J. Rodrigues, and F. Farahmand, "GeoSpray: A geographic routing protocol for vehicular delay-tolerant networks," Inf. Fusion, vol. 15, pp. 102-113, Jan. 2014.

[15] Y.-B. Ko and N. H. Vaidya, "Location-Aided Routing (LAR) in mobile ad hoc networks," ACM Wireless Netw., vol. 6, no. 4, pp. 307-321, Jul. 2000.

[16] V. Erramilli, M. Crovella, A. Chaintreau, and C. Diot, "Delegation forwarding," in Proc. ACM MobiHoc, Hong Kong, May 2008, pp. 251-260.

[17] A. Lindgren, A. Doria, and O. Schelén, "Probabilistic routing in intermittently connected networks," ACM SIGMOBILE Mobile Comput. Commun. Rev., vol. 7, no. 3, pp. 19-20, Jul. 2003.

[18] A. Vahdat and D. Becker, "Epidemic routing for partially-connected ad hoc networks," Duke University, Durham, NC, USA, Tech. Rep. Cs-200006, 2000.

[19] A. Balasubramanian, B. Levine, and A. Venkataramani, "Replication routing in DTNs: A resource allocation approach," IEEE/ACM Trans. Netw., vol. 18, no. 2, pp. 596-609, Apr. 2010.

[20] J. LeBrun, C.-N. Chuah, D. Ghosal, and M. Zhang, "Knowledge-based opportunistic forwarding in vehicular wireless ad hoc networks," in Proc. IEEE VTC-Spring, Stockholm, Sweden, May 2005, pp. 2289-2293.

[21] Y. Cao, Z. Sun, N. Wang, H. Cruickshank, and N. Ahmad, "A reliable and efficient geographic routing scheme for delay/disruption tolerant networks," IEEE Wireless Commun. Lett., vol. 2, no. 6, pp. 603-606, Dec. 2013.

[22] L. Tang, Q. Zheng, J. Liu, and X. Hong, "SMART: A selective controlledflooding routing for delay tolerant networks," in Proc. IEEE BROADNETS, Raleigh, NC, USA, 2007, pp. 356-365.

[23] T. Spyropoulos, K. Psounis, and C. Raghavendra, "Efficient routing in intermittently connected mobile networks: The single-copy case," IEEE/ACM Trans. Netw., vol. 16, no. 1, pp. 63-76, Feb. 2008.

[24] H. Wen et al., "A storage-friendly routing scheme in intermittently connected mobile network," IEEE Trans. Veh. Technol., vol. 60, no. 3, pp. 1138-1149, Mar. 2011.

[25] I. Leontiadis and C. Mascolo, "GeOpps: Geographical opportunistic routing for vehicular networks," in Proc. IEEE WoWMoM, Helsinki, Finland, Feb. 2007, pp. 1-6 
[26] X. Zhang, G. Neglia, J. Kurose, and D. Towsley, "Performance modeling of epidemic routing," Comput. Netw., vol. 51, no. 10, pp. 2867-2891, Jul. 2007.

[27] M. Grossglauser and D. Tse, "Mobility increases the capacity of ad hoc wireless networks," IEEE/ACM Trans. Netw., vol. 10, no. 4, pp. 477-486, Aug. 2002.

[28] A. Keränen, J. Ott, and T. Kärkkäinen, "The ONE simulator for DTN protocol evaluation," in Proc. ICST SIMUTools, Rome, Italy, Mar. 2009, p. 55.

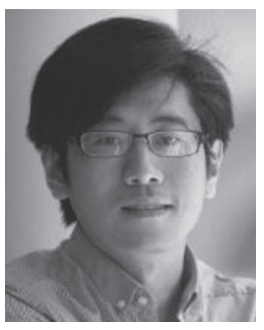

Yue Cao received the Ph.D. degree from the Center for Communication Systems Research (CCSR), University of Surrey, Guildford, U.K., in 2013.

In 2009, he joined the Center for Communication Systems Research, University of Surrey, Guildford, U.K., where he is currently a Research Fellow. His research interests include delay/ disruption-tolerant networks, electric-vehicle communication, and information-centric networking.

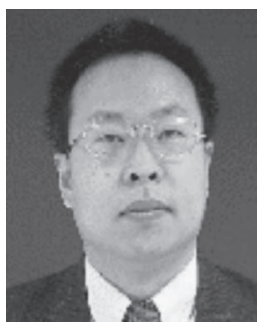

Zhili Sun (M'99) received the Ph.D. degree from Lancaster University, Lancaster, U.K., in 1991.

$\mathrm{He}$ is the Chair of Communication Networking and a Professor with the Center for Communication Systems Research, University of Surrey, Guildford, U.K. His research interests include wireless and sensor networks, satellite communications, mobile operating systems, traffic engineering, Internet protocols and architecture, quality of service, multicast, and security.

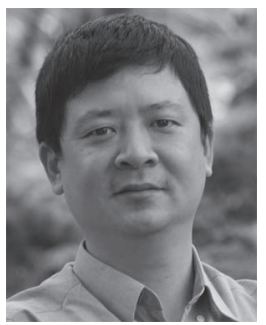

Ning Wang (M'12) received the Ph.D. degree from the University of Surrey, Guildford, U.K., in 2004.

$\mathrm{He}$ is a Senior Lecturer with the Center for Communication Systems Research, University of Surrey. His research interests include network routing optimization, mobility management, traffic engineering, and quality-of-service mechanisms.

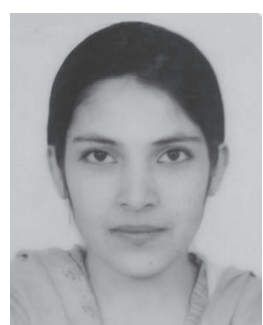

Maryam Riaz is currently working toward the Ph.D. degree with the Center for Communication Systems Research (CCSR), University of Surrey, Guildford, U.K.

She joined the CCSR in 2011. Her research interest includes topology control in mobile ad hoc networks.

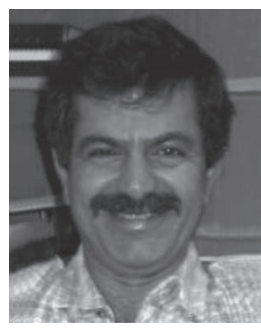

Haitham Cruickshank (M'99) received the Ph.D. degree from Cranfield University, Cranfield, U.K., in 1989.

$\mathrm{He}$ is a Senior Lecturer with the Center for Communication Systems Research, University of Surrey, Guildford, U.K. His main research interests include network security, satellite network architectures, delay-tolerant network security, and qualityof-service provisioning.

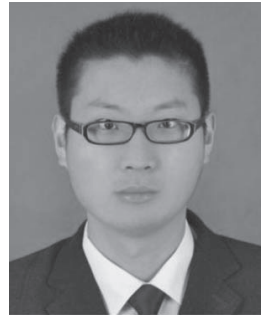

Xiulei Liu received the Ph.D. degree from the State Key Lab of Networking and Switching Technology, Beijing University of Posts and Telecommunications, Beijing, China, in 2012

$\mathrm{He}$ is currently a Postdoctoral Rsearcher with the National Engineering Laboratory for Disaster Backup and Recovery, Beijing University of Posts and Telecommunications. His research interests include semantic web, semantic search, crawler, and ontology. 